\title{
Efficient Approximation of Random Fields for Numerical Applications
}

\author{
Helmut Harbrecht, Michael Peters, \\ Markus Siebenmorgen
}

Institute of Mathematics

Preprint No. 2014-01

University of Basel

January, 2014

Rheinsprung 21

CH - 4051 Basel

Switzerland

$\underline{\text { www.math.unibas.ch }}$ 


\title{
Efficient approximation of random fields for numerical applications $^{\dagger}$
}

\author{
Helmut Harbrecht, Michael Peters*, Markus Siebenmorgen \\ Mathematisches Institut, Rheinsprung 21, 4051 Basel, Switzerland
}

\begin{abstract}
SUMMARY
This article is dedicated to the rapid computation of separable expansions for the approximation of random fields. We consider approaches based on techniques from the approximation of non-local operators on the one hand and based on the pivoted Cholesky decomposition on the other hand. Especially, we provide an a-posteriori error estimate for the pivoted Cholesky decomposition in terms of the trace norm. Numerical examples are provided to validate and quantify the presented methods. Copyright (c) 2014 John Wiley \& Sons, Ltd.

Received ..
\end{abstract}

\section{INTRODUCTION}

In this article, we present and compare two different approaches for the approximation of random fields in $L_{\mathbb{P}}^{2}\left(\Omega, H^{p}(D)\right)$ for a spatial domain $D \in \mathbb{R}^{d}$ and a probability space $(\Omega, \mathcal{F}, \mathbb{P})$. Stochastic fields appear for example in the modeling of diffusion problems with random data, see e.g. [1], and in machine learning, see e.g. [2]. To make a stochastic field $a(\mathbf{x}, \omega)$ feasible for numerical computations in a stochastic Galerkin or stochastic collocation method, see e.g. $[1,3,4,5,6,7]$ and the references therein, one has to separate the spatial variable $\mathbf{x}$ and the stochastic variable $\omega$. Since $L_{\mathbb{P}}^{2}\left(\Omega, H^{p}(D)\right) \cong L_{\mathbb{P}}^{2}(\Omega) \otimes H^{p}(D)$, see e.g. [8], this task can be accomplished by computing a basis representation of $a$ in $L_{\mathbb{P}}^{2}(\Omega) \otimes H^{p}(D)$. A very common approach to obtain such a representation is the Karhunen-Loève expansion, cf. [1,9], which can be regarded as linear operator analogue of the singular value decomposition of matrices.

The main task in the computation of a Karhunen-Loève expansion is the solution of a symmetric and positive semidefinite eigen-problem. In this context, approaches to efficiently compute the Karhunen-Loève expansion have been made by means of the Fast Multipole Method (FMM) based on interpolation, cf. [10], in [11] and with the aid of $\mathcal{H}$-matrices, cf. [12], in [13]. The idea in these articles is to provide a data-sparse representation of the covariance operator which is then used to solve the related eigen-problem numerically by a Krylov subspace method, cf. [14]. Of course, another algorithm for the efficient approximation of non-local operators, like the Adaptive Cross Approximation (ACA), cf. [15, 16], or the Wavelet Galerkin Scheme (WGS), cf. [17, 18], can be considered as well for the representation of the covariance operator. Nevertheless, the major drawback of these approaches is that the number of eigenvalues to be computed has to be known in advance which might be a strong assumption in practice.

\footnotetext{
*Correspondence to: E-mail: michael.peters@unibas.ch

${ }^{\dagger}$ This research has been supported by the Swiss National Science Foundation (SNSF) through the project "Rapid Solution of Boundary Value Problems on Stochastic Domains". 
To overcome this obstruction, we present here an alternative approach based on the Pivoted Cholesky Decomposition (PCD). The PCD can be interpreted as a single-block ACA with applicable total pivoting, cf. [19]. Hence, only the main diagonal of the discretized operator has to be precomputed, which can be performed in essentially, i.e. up to possible poly-logarithmic terms, linear complexity, if the quadrature proposed in [20] is applied to discretize the operator. Then, in each step of the algorithm, the quality of the approximation with respect to the stochastic field is controllable by means of the trace norm. If the desired accuracy is achieved, the algorithm stops with an $M$-term approximation to the operator. If $M$ is substantially smaller than the dimension of the ansatz space, we end up with a remarkable computational speed-up. The related Karhunen-Loève expansion might then be computed in a post-processing step. Notice that then the PCD yields a full but relatively small eigen-problem if the operator under consideration exhibits a certain smoothness. This eigen-problem might be solved numerically exact by e.g. the $Q R$-method, cf. [21].

Now the following question arises: which approach is more efficient? We will try to answer this question numerically by comparing the PCD with methods lend from the approximation of nonlocal operators. We employ here ACA for the data-sparse approximation of the covariance operator which results in a fast matrix-vector product. Thus, a Krylov subspace method - we use the Implicit Restarted Arnoldi Method (IRAM), cf. [22, 23, 24] - is feasible to compute the desired eigenvalues of largest magnitude.

Finally, we would like to emphasize that, although we focus here on the application to random fields, the presented methods are also applicable in the more general case of approximating bivariate functions in $L^{2}\left(D_{1}\right) \otimes L^{2}\left(D_{2}\right)$ for two domains $D_{1} \subset \mathbb{R}^{d_{1}}$ and $D_{2} \subset \mathbb{R}^{d_{2}}$.

The rest of this article is structured as follows. Section 2 is devoted to the Karhunen-Loève expansion. Especially, we discuss here the related error estimates including discretization and truncation error. To that end, it is crucial to have bounds for the decay of the covariance operator's eigenvalues. These bounds are considered here, too. In Section 3, we provide the theoretical background for the pivoted Cholesky decomposition. Moreover, we establish error estimates for the approximation of random fields in terms of the trace norm. These estimates are essential for the a-posteriori control of the approximation error. Section 4 introduces a special class of covariance functions based on the Matérn kernel functions. We choose this class of covariance functions for our numerical tests, since we a-priori know the decay rate of the respective eigenvalues. In particular, we are also able to analytically compute the eigenfunctions and eigenvalues in the case of the unit sphere $\mathbb{S}^{2}$. Thus, these kernels provide an excellent benchmark to compare both approaches. Section 5 is dedicated to testing the numerical performance of the methods under consideration. We will solve the eigenvalue problem for covariance operators related to some of the Matern kernels from Section 4 on different geometries. Finally, we sum up the results presented within this article in Section 6.

In the following, in order to avoid the repeated use of generic but unspecified constants, by $C \lesssim D$ we mean that $C$ can be bounded by a multiple of $D$, independently of parameters which $C$ and $D$ may depend on. Obviously, $C \gtrsim D$ is defined as $D \lesssim C$, and $C \approx D$ as $C \lesssim D$ and $C \gtrsim D$.

\section{THE KARHUNEN-LOÈVE EXPANSION}

Let $(\Omega, \mathcal{F}, \mathbb{P})$ be a probability space with $\sigma$-field $\mathcal{F} \subset 2^{\Omega}$ and a complete probability measure $\mathbb{P}$, i.e. for all $A \subset B$ and $B \in \mathcal{F}$ with $\mathbb{P}[B]=0$ it follows $A \in \mathcal{F}$. Furthermore, let $D \subset \mathbb{R}^{d}$ for $d=2,3$ be a sufficiently smooth and bounded domain.

For $p \geq 0$, the Lebesgue-Bochner space $L_{\mathbb{P}}^{2}\left(\Omega ; H^{p}(D)\right)$ consists of all maps

$$
a: \Omega \rightarrow H^{p}(D)
$$

that satisfy

$$
\|v\|_{L_{\mathbb{P}}^{2}\left(\Omega ; H^{p}(D)\right)}:=\left(\int_{\Omega}\|v(\cdot, \omega)\|_{H^{p}(D)}^{2} \mathrm{~d} \mathbb{P}(\omega)\right)^{1 / 2}<\infty .
$$

In the following, it will be convenient to identify $L_{\mathbb{P}}^{2}\left(\Omega ; H^{p}(D)\right)$ according to

$$
L_{\mathbb{P}}^{2}\left(\Omega ; H^{p}(D)\right) \cong H^{p}(D) \otimes L_{\mathbb{P}}^{2}(\Omega) .
$$


For further details on Lebesgue-Bochner spaces see e.g. [25].

For the approximation of spatial functions in $L^{2}(D)$, we will consider piecewise continuous finite elements. Therefore, we introduce a family of quasi-uniform triangulations $\mathcal{T}_{h}$ for $D$ with mesh width $h$ and define the spaces

$$
V_{h}^{s}:=\left\{v_{h}: D \rightarrow \mathbb{R}:\left.v\right|_{T} \text { is a polynomial of order } s \text { for all } T \in \mathcal{T}_{h}\right\} \subset L^{2}(D) .
$$

Then, given a function $v \in H^{p}(D)$ with $0 \leq p \leq s$, we have due to the Bramble-Hilbert lemma the approximation estimate

$$
\inf _{v_{h} \in V_{h}^{s}}\left\|v-v_{h}\right\|_{L^{2}(D)} \lesssim h^{p}\|v\|_{H^{p}(D)}
$$

uniformly in $h$, see e.g. [26, 27].

A very common representation of random fields for numerical purposes is given by the KarhunenLoève expansion. In order to ensure that $L_{\mathbb{P}}^{2}(\Omega)$ is separable, we have to assume that $\Omega$ is a separable set.

Definition 2.1. Let $a \in H^{p}(D) \otimes L_{\mathbb{P}}^{2}(\Omega)$ for some $p \geq 0$ be a random field. The expansion

$$
a(\mathbf{x}, \omega)=\bar{a}(\mathbf{x})+\sum_{m=1}^{\infty} \sigma_{m} \varphi_{m}(\mathbf{x}) X_{m}(\omega)
$$

with $\sigma_{1} \geq \sigma_{2} \geq \cdots \geq 0,\left(X_{m}, X_{n}\right)_{L_{\mathrm{p}}^{2}(\Omega)}=\delta_{m, n}$ and $\left(\varphi_{m}, \varphi_{n}\right)_{L^{2}(D)}=\delta_{m, n}$ is called KarhunenLoève expansion with respect to a. Here, $\bar{a}(\mathbf{x})$ denotes the mean of a with respect to the stochastic variable, i.e.

$$
\bar{a}(\mathbf{x})=\int_{\Omega} a(\mathbf{x}, \omega) \mathrm{d} \mathbb{P}(\omega) .
$$

The Karhunen-Loève expansion can be regarded as the continuous analogue to the singular value decomposition of matrices. Especially, it holds $\sigma_{m}=\sqrt{\lambda_{m}}$, where $\left\{\left(\lambda_{m}, \varphi_{m}\right)\right\}_{m}$ are the eigen-pairs (in decreasing order) of the covariance operator

$$
(\mathcal{C} u)(\mathbf{x}):=\int_{D} k(\mathbf{x}, \mathbf{y}) u(\mathbf{y}) \mathrm{d} \mathbf{y},
$$

given via the correlation kernel

$$
k(\mathbf{x}, \mathbf{y}):=\int_{\Omega}(a(\mathbf{x}, \omega)-\bar{a}(\mathbf{x}))(a(\mathbf{y}, \omega)-\bar{a}(\mathbf{y})) \mathrm{d} \mathbb{P}(\omega) .
$$

Notice that, for $a \in H^{p}(D) \otimes L_{\mathbb{P}}^{2}(\Omega)$, it holds $k \in H_{\text {mix }}^{p, p}(D \times D):=H^{p}(D) \otimes H^{p}(D)$. Additionally, the random variables $\left\{X_{m}\right\}_{m}$ are given by

$$
X_{m}(\omega)=\frac{1}{\sigma_{m}} \int_{D}(a(\mathbf{x}, \omega)-\bar{a}(\mathbf{x})) \varphi_{m}(\mathbf{x}) \mathrm{d} \mathbf{x} .
$$

In the following, we will also make use of the Hilbert-Schmidt operator associated with the centered random field, i.e. $\mathcal{S}: L_{\mathbb{P}}^{2}(\Omega) \rightarrow H^{p}(D)$ with

$$
(\mathcal{S} u)(\mathbf{x})=\int_{\Omega}(a(\mathbf{x}, \omega)-\bar{a}(\mathbf{x})) u(\omega) \mathrm{d} \mathbb{P}(\omega) \quad \text { for } u \in L_{\mathbb{P}}^{2}(\Omega)
$$

and its adjoint $\mathcal{S}^{\star}: \tilde{H}^{-p}(D) \rightarrow L_{\mathbb{P}}^{2}(\Omega)$ with

$$
\left(\mathcal{S}^{\star} u\right)(\omega)=\int_{D}(a(\mathbf{y}, \omega)-\bar{a}(\mathbf{y})) u(\mathbf{y}) \mathrm{d} \mathbf{y} \quad \text { for } u \in \tilde{H}^{-p}(D) .
$$

Then, we especially find that $\mathcal{S S}^{\star}: \tilde{H}^{-p}(D) \rightarrow H^{p}(D)$ is given by

$$
\left(\mathcal{S S}^{\star} u\right)(\mathbf{x})=\int_{\Omega}(a(\mathbf{x}, \omega)-\bar{a}(\mathbf{x})) \int_{D}(a(\mathbf{y}, \omega)-\bar{a}(\mathbf{y})) u(\mathbf{y}) \mathrm{d} \mathbf{y} \mathrm{d} \mathbb{P}(\omega)=(\mathcal{C} u)(\mathbf{x}),
$$


where the last identity holds due to Fubini's theorem.

For numerical issues, one has to truncate the Karhunen-Loève expansion appropriately. Here, the truncation error depends on the decay of the covariance operator's eigenvalues. More precisely, for the decay of the eigenvalues, we have along the lines of [28] the following theorem.

Theorem 2.2. Let $a \in H^{p}(D) \otimes L_{\mathbb{P}}^{2}(\Omega)$. Then, the eigenvalues of the covariance operator $\mathcal{C}: \tilde{H}^{-p}(D) \rightarrow H^{p}(D)$ decay like

$$
\lambda_{m} \lesssim m^{-2 p / d} \quad \text { for } m \rightarrow \infty .
$$

Proof

We consider the operator $\mathcal{S}^{\star} \mathcal{S}: L_{\mathbb{P}}^{2}(D) \rightarrow L_{\mathbb{P}}^{2}(D)$. Let $\left(\lambda_{m}, X_{m}\right)$ be an eigen-pair of $\mathcal{S}^{\star} \mathcal{S}$. On the one hand, we have

$$
\mathcal{C}\left(\mathcal{S} X_{m}\right)=\lambda_{m}\left(\mathcal{S} X_{m}\right)
$$

On the other hand, it holds for $\lambda_{m}>0$ that

$$
\left(\mathcal{S} X_{m}, \mathcal{S} X_{m}\right)_{L^{2}(D)}=\left(\mathcal{S}^{\star} \mathcal{S} X_{m}, X_{m}\right)_{L_{\mathbb{P}}^{2}(\Omega)}=\lambda_{m}>0 .
$$

Thus, we conclude that $\left(\lambda_{m}, \mathcal{S} X_{m} / \sigma_{m}\right)$ is an eigen-pair of $\mathcal{C}$. The proof is now based on an approximation argument.

We consider the approximation space $V_{h}^{\lceil p\rceil} \subset L^{2}(D)$, cf. (2). Let $\operatorname{dim}\left(V_{h}^{\lceil p\rceil}\right)=N$. Notice that $h \approx N^{-1 / d}$, where the constant depends on the polynomial degree $\lceil p\rceil$. Furthermore, we define the $L^{2}(D)$-orthogonal projection $Q_{N}: L^{2}(D) \rightarrow V_{h}^{\lceil p\rceil}$. Then, due to the Bramble-Hilbert lemma, we conclude the estimate

$$
\left\|\left(I-Q_{N}\right) v\right\|_{L^{2}(D)} \lesssim N^{-p / d}\|v\|_{H^{p}(D)} \quad \text { for } u \in H^{p}(D) .
$$

The min-max principle of Courant-Fisher implies now for arbitrary subspaces $V_{m} \subset L_{\mathbb{P}}^{2}(\Omega)$ with $\operatorname{dim}\left(V_{m}\right) \leq m$ that

$$
\begin{aligned}
\lambda_{m+1} & =\min _{V_{m}} \max _{v \in V_{m}^{\perp},\|v\|_{L_{P}^{2}(\Omega)}=1}\left(\mathcal{S}^{\star} \mathcal{S} v, v\right)_{L_{\mathbb{P}}^{2}(\Omega)} \\
& =\min _{V_{m}} \max _{v \in V_{m}^{\perp},\|v\|_{L_{P}^{2}(\Omega)}=1}(\mathcal{S} v, \mathcal{S} v)_{L^{2}(D) .}
\end{aligned}
$$

For the choice $V_{N}=\operatorname{img}\left(\mathcal{S}^{\star} Q_{N} \mathcal{S}\right)$, the orthogonality of the projection $Q_{N}$ yields

$$
\begin{aligned}
\lambda_{N+1} & \leq \max _{v \perp \operatorname{img}\left(\mathcal{S}^{\star} Q_{N} \mathcal{S}\right),\|v\|_{L_{\mathbb{P}}^{2}(\Omega)}=1}(\mathcal{S} v, \mathcal{S} v)_{L^{2}(D)}\left(\mathcal{S} v,\left(I-Q_{N}\right) \mathcal{S} v\right)_{L^{2}(D)} \\
& =\max _{v \perp \operatorname{img}\left(\mathcal{S}^{\star} Q_{N} \mathcal{S}\right),\|v\|_{L_{\mathbb{P}}^{2}(\Omega)}=1}\left(\left(I-Q_{N}\right) \mathcal{S} v,\left(I-Q_{N}\right) \mathcal{S} v\right)_{L^{2}(D)} \\
& =\max _{v \perp \operatorname{img}\left(\mathcal{S}^{\star} Q_{N} \mathcal{S}\right),\|v\|_{L_{\mathbb{P}}^{2}(\Omega)}=1}\left(\left(I-Q_{N}\right) \mathcal{S} v,\left(I-Q_{N}\right) \mathcal{S} v\right)_{L^{2}(D)} \\
& \leq \sup _{\|v\|_{L_{\mathbb{P}}^{2}(\Omega)}=1}((I-Q \\
& \leq \sup _{\|v\|_{L_{\mathbb{P}}^{2}(\Omega)}=1}\left\|\left(I-Q_{N}\right) \mathcal{S} v\right\|_{L^{2}(D)}^{2} \\
& \lesssim N^{-2 p / d} \sup _{\|v\|_{L_{\mathbb{P}}^{2}(\Omega)}=1}\|\mathcal{S} v\|_{H^{p}(D)}
\end{aligned}
$$

This estimate together with the continuity of $\mathcal{S}$, see e.g. [28], yields the assertion.

Now, in accordance with [28], an estimation of the Karhunen-Loève expansion's truncation error is provided by the following theorem. 
Theorem 2.3. Let $a \in H^{p}(D) \otimes L_{\mathbb{P}}^{2}(\Omega)$ with $p>d / 2$. Then, it holds

$$
\left\|a-\bar{a}-\sum_{m=1}^{M} \sigma_{m}\left(\varphi_{m} \otimes X_{m}\right)\right\|_{L^{2}(D) \otimes L_{\mathbb{P}}^{2}(\Omega)}=\sqrt{\sum_{m=M}^{\infty} \lambda_{m}} \lesssim M^{\frac{1}{2}-\frac{p}{d}} .
$$

Proof

For a proof of this theorem see [28].

This theorem tells us that, in order to guarantee an error bound

$$
\left\|a-\bar{a}-\sum_{m=1}^{M} \sigma_{m}\left(\varphi_{m} \otimes X_{m}\right)\right\|_{L^{2}(D) \otimes L_{\mathbb{P}}^{2}(\Omega)} \lesssim \varepsilon,
$$

we have to choose

$$
M \approx \varepsilon^{\frac{2 d}{d-2 p}} .
$$

For the numerical purposes, we have also to take the smoothness of the covariance operator's eigenfunctions into account, $\mathrm{cf}$. [28, 11].

Theorem 2.4. Let $a \in H^{p}(D) \otimes L_{\mathbb{P}}^{2}(\Omega)$. Then, the eigenfunctions $\left\{\varphi_{m}\right\}_{m}$ of the covariance operator $\mathcal{C}$ satisfy

$$
\left\|\varphi_{m}\right\|_{H^{t}(D)} \lesssim \sigma_{m}^{-\frac{t}{p}}, \quad 0 \leq t \leq p .
$$

Proof

From (6) and the continuity of $\mathcal{S}$ we deduce

$$
\left\|\varphi_{m}\right\|_{H^{p}(D)}=\frac{1}{\sigma_{m}}\left\|\mathcal{S} X_{m}\right\|_{H^{p}(D)} \lesssim \frac{1}{\sigma_{m}}\left\|X_{m}\right\|_{L_{P}^{2}(\Omega)}=\frac{1}{\sigma_{m}} .
$$

By using in addition $\left\|\varphi_{m}\right\|_{L^{2}(D)}=1$, we obtain the assertion via an interpolation argument.

Remark 2.5. As a consequence of Theorem 2.4, we can approximate the eigenfunctions in $V_{h}^{s}$ according to

$$
\inf _{v_{h} \in V_{h}^{s}}\left\|\varphi_{m}-v_{h}\right\|_{L^{2}(D)} \lesssim h^{s}\left\|\varphi_{m}\right\|_{H^{s}(D)} \lesssim \sigma_{m}^{-\frac{s}{p}} h^{s} .
$$

The approximation of the related eigenvalues is given in terms of the gap between the invariant sub-space $U_{m}=\operatorname{span}\left\{\varphi_{1}, \ldots, \varphi_{m}\right\} \subset L^{2}(D)$ corresponding to the eigenvalues $\lambda_{1}, \ldots, \lambda_{m}$ and the approximation space $V_{h}^{s}$, i.e.

$$
\theta\left(U_{m}\right):=\sup _{u \in U_{m},\|u\|_{L^{2}(D)}=1}\left\|\left(I-Q_{h}\right) u\right\|_{L^{2}(D)},
$$

where $Q_{h}: L^{2}(D) \rightarrow V_{h}^{s}$ denotes the $L^{2}(D)$ orthogonal projection onto $V_{h}^{s}$.

Lemma 2.6. Let $U_{m}=\operatorname{span}\left\{\varphi_{1}, \ldots, \varphi_{m}\right\} \subset L^{2}(D)$ be the invariant sub-space corresponding to the eigenvalues $\lambda_{1}, \ldots, \lambda_{m}$. Then, it holds

$$
\theta\left(U_{m}\right) \lesssim \sigma_{m}^{-\frac{s}{p}} h^{s}
$$

Proof

Let $u=\sum_{i=1}^{m} \alpha_{i} \varphi_{i}$. Thus, it holds $\sum_{i=1}^{m} \alpha_{i}^{2}=1$ since $\|u\|_{L^{2}(D)}=1$. Then, with $\boldsymbol{\alpha}=$ $\left(\alpha_{1}, \ldots, \alpha_{m}\right)$, we have

$$
\begin{aligned}
\theta\left(U_{m}\right) & =\sup _{u \in U_{m},\|u\|_{L^{2}(D)}=1}\left\|\left(I-Q_{h}\right) u\right\|_{L^{2}(D)}=\sup _{\|\boldsymbol{\alpha}\|_{\ell^{2}}=1}\left\|\sum_{i=1}^{m} \alpha_{i}\left(I-Q_{h}\right) \varphi_{i}\right\|_{L^{2}(D)} \\
& \leq \sup _{\|\boldsymbol{\alpha}\|_{\ell^{2}}=1} \sum_{i=1}^{m}\left|\alpha_{i}\right|\left\|\left(I-Q_{h}\right) \varphi_{i}\right\|_{L^{2}(D)} \lesssim \sup _{\|\boldsymbol{\alpha}\|_{\ell^{2}}=1} \sum_{i=1}^{m}\left|\alpha_{i}\right| \sigma_{i}^{-\frac{s}{p}} h^{s} \\
& \lesssim \sigma_{m}^{-\frac{s}{p}} h^{s}
\end{aligned}
$$

where we used (7) in the second to last step. 
Remark 2.7. In order to achieve convergence for the $m$-th eigenvalue, we have to guarantee $\theta\left(U_{m}\right)<1$ which imposes a restriction to the mesh width $h$ of the discretization.

From [29], we have the following convergence result, which relates the eigenvalue's rate of approximation to the eigenfunction's rate of approximation.

Theorem 2.8. Let $\left\{\left(\lambda_{m}, \varphi_{m}\right)\right\}_{m}$ be the set of eigen-pairs of the covariance operator $\mathcal{C}$ as defined in (5). Furthermore, let $U_{M}=\operatorname{span}\left\{\varphi_{1}, \ldots, \varphi_{M}\right\}$ be such that $\operatorname{dim}\left(Q_{h} U_{M}\right)=M$. Then, the approximation $\lambda_{m, h}$ to the $m$-th eigenvalue by the Rayleigh-Ritz method, i.e. $Q_{h} \mathcal{C} Q_{h} \varphi_{m, h}=$ $\lambda_{m, h} \varphi_{m, h}$, satisfies the estimate

$$
0 \leq \lambda_{m}-\lambda_{m, h} \leq \lambda_{m}\left(\theta\left(U_{m}\right)\right)^{2} \quad \text { for all } 1 \leq m \leq M .
$$

Proof

The proof of this theorem can be found in [29].

By inserting (8) into (9), we thus arrive at

$$
0 \leq \lambda_{m}-\lambda_{m, h} \lesssim \lambda_{m}^{\frac{p-s}{p}} h^{2 s} .
$$

Hence, given that the random variables $\left\{X_{m}\right\}_{m}$ in the Karhunen-Loève expansion are represented exactly, we finally derive the following result for the approximation of the stochastic field $a$ by the discretized truncated Karhunen-Loève expansion.

Theorem 2.9. Let the length $M$ of the Karhunen-Loève expansion be such that the truncation error is smaller than $\varepsilon$ for some $\varepsilon>0$. Then, we have

$$
\left\|a-\bar{a}_{h}-\sum_{m=1}^{M} \sigma_{m, h}\left(\varphi_{m, h} \otimes X_{m}\right)\right\|_{L^{2}(D) \otimes L_{\mathbb{P}}^{2}(\Omega)} \lesssim \varepsilon+c(M) h^{s}
$$

with a constant $c(M) \leq M$ which might depend on $s, p, d$, but is independent of $h$.

Proof

Since the random variables are exactly represented, we have by splitting up the error and using Theorem 2.3 that

$$
\begin{aligned}
& \left\|a-\bar{a}_{h}-\sum_{m=1}^{M} \sigma_{m, h}\left(\varphi_{m, h} \otimes X_{m}\right)\right\|_{L^{2}(D) \otimes L_{\mathbb{P}}^{2}(\Omega)} \\
& \quad \lesssim \varepsilon+\left\|\bar{a}-\bar{a}_{h}\right\|_{L^{2}(D)}+\left\|\sum_{m=1}^{M}\left(\sigma_{m} \varphi_{m}-\sigma_{m, h} \varphi_{m, h}\right) \otimes X_{m}\right\|_{L^{2}(D) \otimes L_{\mathbb{P}}^{2}(\Omega)} \\
& \quad \lesssim \varepsilon+h^{s}\|\bar{a}\|_{H^{s}(D)}+\sum_{m=1}^{M}\left\|\sigma_{m} \varphi_{m}-\sigma_{m, h} \varphi_{m, h}\right\|_{L^{2}(D)},
\end{aligned}
$$

where we used in the last step that $\left\|X_{m}\right\|_{L_{\mathrm{p}}^{2}(\Omega)}=1$. Each summand in the last term is now estimated as follows:

$$
\begin{aligned}
\left\|\sigma_{m} \varphi_{m}-\sigma_{m, h} \varphi_{m, h}\right\|_{L^{2}(D)} & \leq\left\|\left(\sigma_{m}-\sigma_{m, h}\right) \varphi_{m}\right\|_{L^{2}(D)}+\left\|\sigma_{m, h}\left(\varphi_{m}-\varphi_{m, h}\right)\right\|_{L^{2}(D)} \\
& \lesssim \sigma_{m}-\sigma_{m, h}+\sigma_{m, h} \sigma_{m}^{-\frac{s}{p}} h^{s},
\end{aligned}
$$

due to $\left\|\varphi_{m}\right\|_{L^{2}(D)}=1$ and the estimate of the gap in Lemma 2.6. Furthermore, we have for the singular values

$$
\left(\sigma_{m}-\sigma_{m, h}\right)^{2}=\lambda_{m}-2 \sigma_{m} \sigma_{m, h}+\lambda_{m, h} \leq \lambda_{m}-\lambda_{m, h} \lesssim \lambda_{m}^{\frac{p-s}{p}} h^{2 s},
$$


since $\sigma_{m, h} \leq \sigma_{m}$, and therefore

$$
\left\|\sigma_{m} \varphi_{m}-\sigma_{m, h} \varphi_{m, h}\right\|_{L^{2}(D)} \lesssim \sigma_{m}^{\frac{p-s}{p}} h^{s} .
$$

Inserting this in the above estimate yields together with Theorem 2.2 that

$$
\begin{aligned}
\left\|a-\bar{a}_{h}-\sum_{m=1}^{M} \sigma_{m, h}\left(\varphi_{m, h} \otimes X_{m}\right)\right\|_{L^{2}(D) \otimes L_{\mathbb{P}}^{2}(\Omega)} & \lesssim \varepsilon+h^{s}\|\bar{a}\|_{H^{s}(D)}+\sum_{m=1}^{M} \sigma_{m}^{\frac{p-s}{p}} h^{s} \\
& \lesssim \varepsilon+h^{s}\|\bar{a}\|_{H^{s}(D)}+h^{s} \sum_{m=1}^{M} m^{\frac{s-p}{d}} .
\end{aligned}
$$

Estimating the sum by the respective integral yields

$$
c(M)=\sum_{m=1}^{M} m^{\frac{s-p}{d}} \approx \int_{1}^{M} x^{\frac{s-p}{d}} \mathrm{~d} x= \begin{cases}\frac{d}{s-p+d}\left(M^{\frac{s-p+d}{d}}-1\right), & \text { for } s \neq p-d \\ \log (M), & \text { else }\end{cases}
$$

This completes the proof.

\section{THE PIVOTED CHOLESKY DECOMPOSITION}

In this section, we consider an alternative approach for the representation of a random field. This approach makes use of the pivoted Cholesky decomposition as considered in [19]. The idea is here to approximate the spatially discretized stochastic field $Q_{h} a$ rather than to approximate the stochastic field itself. One easily verifies for $a \in H^{p}(D) \otimes L_{\mathbb{P}}^{2}(\Omega)$ that

$$
\left\|\left(I-Q_{h}\right) a\right\|_{L^{2}(D) \otimes L_{\mathbb{P}}^{2}(\Omega)} \leq h^{s}\|a\|_{H^{p}(D) \otimes L_{\mathbb{P}}^{2}(\Omega)} \quad \text { for } 0 \leq s \leq p
$$

by the definition of the Bochner norms (1) and the estimate (3). Furthermore, due to Fubini's theorem, we conclude for the mean that

$$
\int_{\Omega} Q_{h} a(\mathbf{x}, \omega) \mathrm{d} \mathbb{P}(\omega)=Q_{h} \bar{a}(\mathbf{x})
$$

The related covariance operator is then given by

$$
\begin{aligned}
\left(\mathcal{C}_{h} u\right)(\mathbf{x}) & =\int_{D} \int_{\Omega} Q_{h, \mathbf{x}}(a(\mathbf{x}, \omega)-\bar{a}(\mathbf{x})) Q_{h, \mathbf{y}}(a(\mathbf{y}, \omega)-\bar{a}(\mathbf{y})) \mathrm{d} \mathbb{P}(\omega) u(\mathbf{y}) \mathrm{d} \mathbf{y} \\
& =Q_{h, \mathbf{x}} \int_{D} Q_{h, \mathbf{y}} k(\mathbf{x}, \mathbf{y}) u(\mathbf{y}) \mathrm{d} \mathbf{y} \\
& =Q_{h, \mathbf{x}} \int_{D} k(\mathbf{x}, \mathbf{y}) Q_{h, \mathbf{y}} u(\mathbf{y}) \mathrm{d} \mathbf{y} \\
& =\left(Q_{h} \mathcal{C} Q_{h} u\right)(\mathbf{x}) .
\end{aligned}
$$

Especially, for each finite dimensional ansatz space, $\mathcal{C}_{h}$ is a symmetric and positive semidefinite matrix. Thus, $\mathcal{C}_{h}$ exhibits a (possibly pivoted) Cholesky decomposition. By pivoting the Cholesky 
decomposition, cf. Algorithm 1, we achieve numerical stability on the one hand, cf. [30], and, if the eigenvalues of $\mathcal{C}_{h}$ decay sufficiently fast, a low-rank approximation on the other hand, cf. [19].

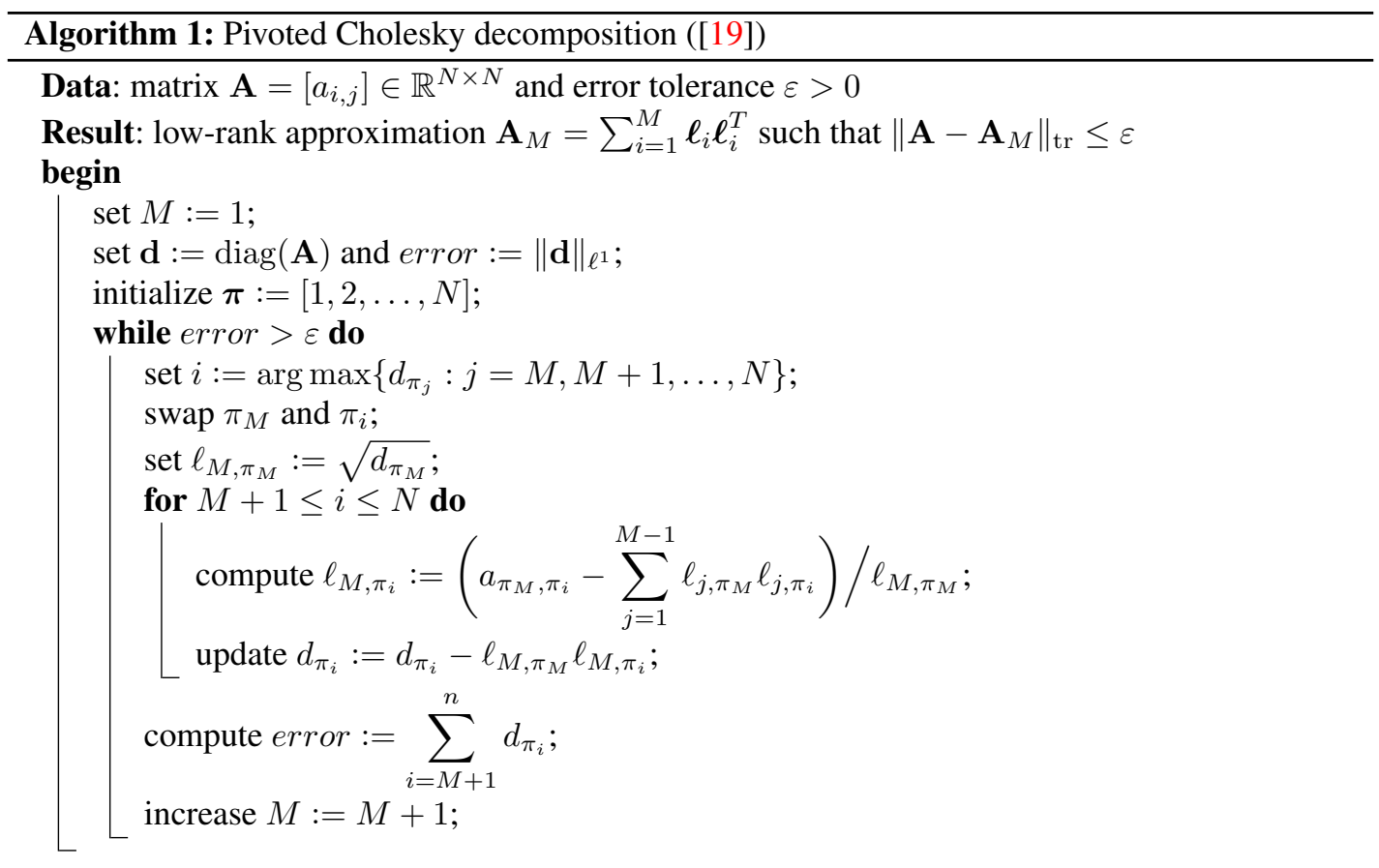

The approximation error of the (truncated) pivoted Cholesky decomposition is a-posteriori controllable in terms of the trace norm, i.e.

$$
\|\mathbf{A}\|_{\text {tr }}:=\operatorname{trace}(\mathbf{A}):=\sum_{i=1}^{N} a_{i, i} .
$$

Suppose that the pivoted Cholesky decomposition terminates with an approximation $\mathcal{C}_{h, M}$ to $\mathcal{C}_{h}$ with

$$
\left\|\mathcal{C}_{h}-\mathcal{C}_{h, M}\right\|_{\text {tr }}<\varepsilon .
$$

We denote the spectral decompositions related to $\mathcal{C}_{h}$ by

$$
\mathcal{C}_{h}=\sum_{i=1}^{N} \lambda_{i} \mathbf{v}_{i} \mathbf{v}_{i}^{\top}
$$

With respect to the orthonormal basis $\mathbf{\Phi}(\mathbf{x}):=\left[\phi_{1}(\mathbf{x}), \ldots, \phi_{N}(\mathbf{x})\right]$ of $V_{h}^{s}$, the Karhunen-Loève expansion of $a_{h}(\mathbf{x}, \omega)$ is then given by

$$
a_{h}(\mathbf{x}, \omega)=\boldsymbol{\Phi}(\mathbf{x}) \overline{\mathbf{a}}_{h}+\sum_{i=1}^{N} \sqrt{\lambda_{i}} \boldsymbol{\Phi}(\mathbf{x}) \mathbf{v}_{i} X_{i}(\omega) .
$$

This representation can be rewritten in matrix notation as

$$
a_{h}(\mathbf{x}, \omega)-\mathbf{\Phi}(\mathbf{x}) \overline{\mathbf{a}}_{h}=: \mathbf{\Phi}(\mathbf{x}) \mathbf{V} \mathbf{\Sigma} \mathbf{X}(\omega)
$$

with $\mathbf{V}:=\left[\mathbf{v}_{1}, \ldots, \mathbf{v}_{N}\right], \boldsymbol{\Sigma}:=\operatorname{diag}\left(\sqrt{\lambda_{1}}, \ldots, \sqrt{\lambda_{N}}\right)$ and $\mathbf{X}(\omega):=\left[X_{1}(\omega), \ldots, X_{N}(\omega)\right]^{\top}$.

The matrix $(\mathbf{V} \boldsymbol{\Sigma})^{\top} \in \mathbb{R}^{N \times N}$ from (16) exhibits a QR-decomposition:

$$
\mathbf{Q L}^{\top}=(\mathbf{V} \boldsymbol{\Sigma})^{\top} \text { or } \mathbf{L} \mathbf{Q}^{\top}=\mathbf{V} \boldsymbol{\Sigma},
$$


respectively. Here, $\mathbf{Q} \in \mathbb{R}^{N \times N}$ denotes an orthogonal matrix, i.e. $\mathbf{Q}^{\top} \mathbf{Q}=\mathbf{I} \in \mathbb{R}^{N \times N}$, and $\mathbf{L} \in$ $\mathbb{R}^{N \times N}$ is a lower triangular matrix. We shall next define the transformed random vector

$$
\mathbf{Y}(\omega):=\mathbf{Q}^{\top} \mathbf{X}(\omega)
$$

Then, $\mathbf{Y}(\omega)$ also consists of $N$ uncorrelated and centered random variables, since it holds

$$
\int_{\Omega} \mathbf{Y}(\omega) \mathbf{Y}^{\boldsymbol{\top}}(\omega) \mathrm{d} \mathbb{P}(\omega)=\mathbf{Q}^{\boldsymbol{\top}} \int_{\Omega} \mathbf{X}(\omega) \mathbf{X}^{\boldsymbol{\top}}(\omega) \mathrm{d} \mathbb{P}(\omega) \mathbf{Q}=\mathbf{Q}^{\boldsymbol{\top}} \mathbf{I} \mathbf{Q}=\mathbf{I} .
$$

That the random variables $Y_{i}(\omega)$ are also centered, follows from the fact that they are weighted sums of centered random variables. Thus, we obtain for the covariance matrix $\mathcal{C}_{h}$ of $a_{h}(\mathbf{x}, \omega)$ that

$$
\mathcal{C}_{h}=\int_{\Omega}(\mathbf{V} \boldsymbol{\Sigma} \mathbf{X}(\omega))(\mathbf{V} \boldsymbol{\Sigma} \mathbf{X}(\omega))^{\top} \mathrm{d} \mathbb{P}(\omega)=\int_{\Omega} \mathbf{L Y}(\omega) \mathbf{Y}(\omega)^{\top} \mathbf{L}^{\top} \mathrm{d} \mathbb{P}(\omega)=\mathbf{L L}^{\top} .
$$

Since $\mathbf{L}$ is a lower triangular matrix, we thus end up with the Cholesky decomposition of $\mathcal{C}_{h}$. In the following, without loss of generality, we will especially assume, that $\mathbf{L} \mathbf{L}^{\top}$ corresponds to the pivoted Cholesky decomposition of $\mathcal{C}_{h}$.

Using the Cholesky decomposition of $\mathcal{C}_{h}$, we obtain the separable representation

$$
a_{h}(\mathbf{x}, \omega)=\boldsymbol{\Phi}(\mathbf{x}) \overline{\mathbf{a}}_{h}+\sum_{i=1}^{N} \boldsymbol{\Phi}(\mathbf{x}) \boldsymbol{\ell}_{i} Y_{i}(\omega)=\boldsymbol{\Phi}(\mathbf{x}) \overline{\mathbf{a}}_{h}+\boldsymbol{\Phi}(\mathbf{x}) \mathbf{L Y}(\omega)
$$

for $a(\mathbf{x}, \omega)$ with $\mathbf{L}=\left[\ell_{1}, \ldots, \ell_{N}\right]$. Whereas, the related truncated Cholesky decomposition leads to the truncated expansion

$$
a_{h, M}(\mathbf{x}, \omega)=\boldsymbol{\Phi}(\mathbf{x}) \overline{\mathbf{a}}_{h}+\sum_{i=1}^{M} \mathbf{\Phi}(\mathbf{x}) \ell_{i} Y_{i}(\omega) .
$$

It is easy to see that $\mathcal{C}_{h, M}$ is the covariance matrix of $a_{h, M}(\mathbf{x}, \omega)$.

Remark 3.1. The separable representation (17) of the stochastic field is based on the knowledge of an appropriate matrix $\mathbf{R} \in \mathbb{R}^{N \times N}$, a square root of the covariance matrix, such that $\mathcal{C}_{h}=$ $\mathbf{R R}^{\top}$. It is known that for two different square roots, i.e. $\mathcal{C}_{h}=\mathbf{R R}^{\top}=\tilde{\mathbf{R}} \tilde{\mathbf{R}}^{\top}$, there exists an orthogonal matrix $\mathbf{Q} \in \mathbb{R}^{N \times N}$ such that $\tilde{\mathbf{R}}=\mathbf{R Q}^{\top}$. The change of the representation (16) due to the application of $\mathbf{Q}$ is then performed by the change of the basis in $L_{\mathbb{P}}^{2}(\Omega)$, i.e. $\mathbf{Y}(\omega):=\mathbf{Q}^{\top} \mathbf{X}(\omega)$. Thus, any square root of $\mathcal{C}_{h}$ yields a separable representation of $a_{h}(\mathbf{x}, \omega)$. Nevertheless, we focus on the pivoted Cholesky decomposition here.

The approximation error of a given stochastic field by truncation of the pivoted Cholesky decomposition is now controllable in accordance with the following theorem.

Theorem 3.2. For a given covariance matrix $\mathcal{C}_{h} \in \mathbb{R}^{N \times N}$ given by (13), let $\mathcal{C}_{h, M} \in \mathbb{R}^{N \times N}$ denote its pivoted Cholesky decomposition computed by Algorithm 1 such that

$$
\left\|\mathcal{C}_{h}-\mathcal{C}_{h, M}\right\|_{\text {tr }}<\varepsilon
$$

holds for some $\varepsilon>0$. Then, for the related stochastic fields, we have the error estimate

$$
\left\|a_{h}-a_{h, M}\right\|_{L^{2}(D) \otimes L_{\mathbb{P}}^{2}(\Omega)}<\sqrt{\varepsilon} .
$$

Proof

Let $\mathcal{C}_{h, M}=\mathbf{L}_{M} \mathbf{L}_{M}^{\top}$ be the pivoted Cholesky decomposition computed by Algorithm 1. We denote the (pivoted) Cholesky decomposition of the Schur-complement $\mathcal{C}_{h}-\mathcal{C}_{h, M}$ by $\mathbf{E}$, that is $\mathcal{C}_{h}-\mathcal{C}_{h, M}=\mathbf{E E}^{\top}$ with a lower triangular matrix $\mathbf{E} \in \mathbb{R}^{N \times N}$ with $\mathbf{E}=\left[\mathbf{e}_{1}, \ldots, \mathbf{e}_{N}\right]$. Then, it holds

$$
\mathbf{L L}^{\top}=\left(\mathbf{L}_{M}+\mathbf{E}\right)\left(\mathbf{L}_{M}+\mathbf{E}\right)^{\top}
$$


and therefore

$$
\mathbf{E}=\mathbf{L}-\mathbf{L}_{M} .
$$

Noticing that $a_{h, M}(\mathbf{x}, \omega)=\mathbf{\Phi} \mathbf{L}_{M} \mathbf{Y}(\omega)$, we are able to estimate the error of the approximate random field according to

$$
\begin{aligned}
\left\|a_{h}-a_{h, M}\right\|_{L^{2}(D) \otimes L_{\mathbb{P}}^{2}(\Omega)}^{2} & =\left\|\mathbf{\Phi} \mathbf{L} \mathbf{Y}-\mathbf{\Phi} \mathbf{L}_{M} \mathbf{Y}\right\|_{L^{2}(D) \otimes L_{\mathbb{P}}^{2}(\Omega)}^{2}=\|\mathbf{\Phi} \mathbf{E} \mathbf{Y}\|_{L^{2}(D) \otimes L_{\mathbb{P}}^{2}(\Omega)}^{2} \\
& =\int_{D} \int_{\Omega}\left(\sum_{i=M+1}^{N} \mathbf{\Phi}(\mathbf{x}) \boldsymbol{\ell}_{i} Y_{i}(\omega)\right)^{2} \mathrm{~d} \mathbb{P}(\omega) \mathrm{d} \mathbf{x} \\
& =\int_{D} \sum_{i=M+1}^{N}\left(\mathbf{\Phi}(\mathbf{x}) \boldsymbol{\ell}_{i}\right)^{2} \mathrm{~d} \mathbf{x}=\sum_{i=M+1}^{N} \int_{D}\left(\mathbf{\Phi}(\mathbf{x}) \boldsymbol{\ell}_{i}\right)^{2} \mathrm{~d} \mathbf{x} \\
& =\sum_{i=M+1}^{N} \boldsymbol{\ell}_{i}^{\top} \boldsymbol{\ell}_{i}=\sum_{i=1}^{N} \mathbf{e}_{i}^{\top} \mathbf{e}_{i} \\
& =\operatorname{trace}\left(\mathbf{E E}^{\top}\right)<\varepsilon .
\end{aligned}
$$

The theorem states that the choice $\varepsilon \approx h^{2 s}$ in the pivoted Cholesky decomposition guarantees, together with inequality (12), the error estimate

$$
\left\|a-a_{h, M}\right\|_{L^{2}(D) \otimes L_{\mathbb{P}}^{2}(\Omega)} \lesssim h^{s} .
$$

The major advantage of this approach is, that at no time the covariance matrix $\mathcal{C}_{h}$ has to be fully assembled. It is sufficient to provide access to single entries of this matrix while processing the pivoted Cholesky decomposition. The error in the approximation of the random field $a$ is then aposteriori controllable by the trace norm. Furthermore, it is shown in [19] that the pivoted Cholesky decomposition is optimal in the sense of best $M$-term approximations for sufficiently fast decaying eigenvalues.

Given that the pivoted Cholesky decomposition for $\mathcal{C}_{h}$ truncates with $M \ll N$ terms and $\mathcal{C}_{h, M}=$ $\mathbf{L}_{M} \mathbf{L}_{M}^{\top} \in \mathbb{R}^{N \times N}$, the computation of the related Karhunen-Loève expansion is performed with complexity $\mathcal{O}\left(M^{2} N\right)$, cf. [19]. This can be achieved by computing the eigenvalues of $\mathbf{L}_{M}^{\top} \mathbf{L}_{M} \in$ $\mathbb{R}^{M \times M}$ which coincide with those of $\mathcal{C}_{h, M}$. Then, if $\mathbf{v}_{1}, \ldots, \mathbf{v}_{M}$ denote the orthonormal vectors of the small eigen-problem, the eigenvectors of $\mathcal{C}_{h, M}$ are given by $\mathbf{L v}_{1}, \ldots, \mathbf{L v}_{M}$ and we have

$$
\left(\mathbf{L v}_{i}\right)^{\top}\left(\mathbf{L v}_{j}\right)=\mathbf{v}_{i} \mathbf{L}^{\top} \mathbf{L} \mathbf{v}_{j}=\lambda_{i} \delta_{i, j} \quad \text { for all } i, j=1, \ldots, M .
$$

Thus, the related Karhunen-Loève decomposition is given by

$$
a_{h, M}(\mathbf{x}, \omega)=\bar{a}_{h}(\mathbf{x})+\sum_{i=1}^{M} \boldsymbol{\Phi}(\mathbf{x}) \mathbf{L v}_{i} \tilde{X}_{i}(\omega) .
$$

If the laws of the random variables $X_{i}(\omega)$ are known, we obtain the relation

$$
\tilde{\mathbf{X}}(\omega)=\left[\mathbf{v}_{1}, \ldots, \mathbf{v}_{M}\right]^{\top} \mathbf{Y}(\omega)
$$

with $\mathbf{v}_{i}$ from (19). Otherwise, the related random variables $\tilde{X}_{i}(\omega)$ can be determined by a maximum likelihood estimate, cf. [11]. Notice that for the important Gaussian case $\mathbf{X}(\omega) \sim[\mathcal{N}(0,1)]^{N}$, we have for any orthogonal transform $\mathbf{Q X}(\omega) \sim[\mathcal{N}(0,1)]^{N}$ and thus $\tilde{\mathbf{X}}$ has the same law as $\mathbf{X}$.

\section{THE MARTÉRN CLASS OF KERNELS}

For our numerical tests, we consider a special subset of Hilbert-Schmidt kernels, namely the Matérn class of kernel functions, cf. [31]. They are very often used as covariance kernels for the definition of stochastic fields. In accordance with [2], they are defined as follows. 
Definition 4.1. Let $r:=\|\mathbf{x}-\mathbf{y}\|_{2}$ and $\ell \in(0, \infty)$. Then, the Matérn covariance function of order $\nu>0$ is given by

$$
k_{\nu}(r):=\frac{2^{1-\nu}}{\Gamma(\nu)}\left(\frac{\sqrt{2 \nu} r}{\ell}\right)^{\nu} K_{\nu}\left(\frac{\sqrt{2 \nu} r}{\ell}\right) .
$$

Here, $\Gamma$ denotes the gamma function and $K_{\nu}$ denotes the modified Bessel function of the second kind of order $\nu, c f .[32]$.

The expression (20) simplifies if $\nu=p+1 / 2$ with $p \in \mathbb{N}$. In this case, [2] provides

$$
k_{p+1 / 2}(r)=\exp \left(-\frac{\sqrt{2 \nu} r}{\ell}\right) \frac{p !}{(2 p) !} \sum_{i=0}^{p} \frac{(p+i) !}{i !(p-i) !}\left(\frac{\sqrt{8 \nu} r}{\ell}\right)^{p-i}
$$

Especially, we have

$$
\begin{aligned}
\nu & =\frac{1}{2}, & k_{1 / 2}(r) & =\exp \left(-\frac{r}{\ell}\right), \\
\nu & =\frac{3}{2}, & k_{3 / 2}(r) & =\left(1+\frac{\sqrt{3} r}{\ell}\right) \exp \left(-\frac{\sqrt{3} r}{\ell}\right), \\
\nu & =\frac{5}{2}, & k_{5 / 2}(r) & =\left(1+\frac{\sqrt{5} r}{\ell}+\frac{5 r^{2}}{3 \ell^{2}}\right) \exp \left(-\frac{\sqrt{5} r}{\ell}\right), \\
\nu & =\frac{7}{2}, & k_{7 / 2}(r) & =\left(1+\frac{\sqrt{7} r}{\ell}+\frac{14 r^{2}}{5 \ell^{2}}+\frac{49 \sqrt{7} r^{3}}{15 \ell^{3}}\right) \exp \left(-\frac{\sqrt{7 r}}{\ell}\right), \\
\nu & =\frac{9}{2}, & k_{9 / 2}(r) & =\left(1+\frac{3 r}{\ell}+\frac{27 r^{2}}{7 \ell^{2}}+\frac{18 r^{3}}{7 \ell^{3}}+\frac{27 r^{4}}{35 \ell^{3}}\right) \exp \left(-\frac{3 r}{\ell}\right), \\
\nu & =\infty, & k_{\infty}(r) & =\exp \left(-\frac{r^{2}}{2 \ell^{2}}\right) .
\end{aligned}
$$

A visualization of this kernels for different values of $\nu$ is given in Figure 1. Obviously, the Sobolev smoothness of the kernel $k_{\nu}$ is controlled by the smoothness parameter $\nu$.

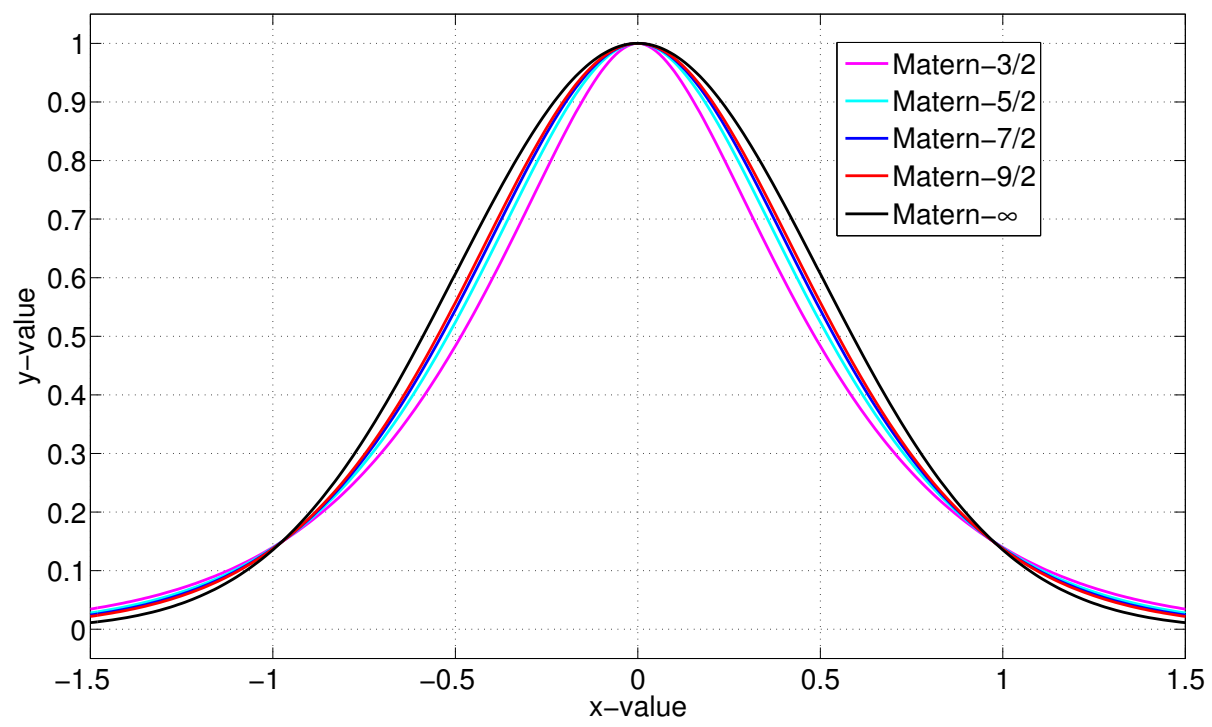

Figure 1. Different values for the smoothness parameter $\nu$. 
For increasing values of $\nu$, the respective kernel function $k_{\nu}$ exhibits successively more regularity. Especially, the eigenvalues of the Matérn correlation kernels decay like

$$
\lambda_{m} \leq C m^{-\left(1+\frac{2 \nu}{d}\right)}
$$

for some $C>0$, cf. [33]. Thus, since the decay of the covariance operator's eigenvalues is known in advance, they are very well suited for numerical examples.

Obviously, the Matérn kernels provide rotational symmetry, i.e. they are invariant under isometries of $D$, since they are only dependent on the particular distance of the points $\mathbf{x}$ and $\mathbf{y}$. Thus, we obtain analytic expressions for the eigenvalues of the underlying Hilbert-Schmidt operators, if we choose $D=\mathbb{S}^{d-1}$ to be the $d$-dimensional unit sphere. More precisely, we may apply the FunkHecke formula, cf. [34], which reads as follows.

Theorem 4.2. Let $\mathrm{x} \in \mathbb{S}^{d-1}$ and $f \in C([-1,1])$, then it holds

$$
\int_{\mathbb{S}^{d-1}} f\left(\mathbf{x}^{\top} \mathbf{y}\right) Y_{m}(\mathbf{y}) \mathrm{d} \sigma_{\mathbf{y}}=\lambda_{m} Y_{m}(\mathbf{x})
$$

with

$$
\lambda_{m}=\left|\mathbb{S}^{d-2}\right| \int_{-1}^{1} P_{m}(d ; t) f(t)\left(1-t^{2}\right)^{\frac{d-3}{2}} \mathrm{~d} t .
$$

Here, $Y_{m}$ corresponds to a spherical harmonic of order $m$ and $P_{m}(d ; t)$ denotes the polynomial

$$
P_{m}(d ; t):=m ! \Gamma\left(\frac{d-1}{2}\right) \sum_{i=0}^{\lfloor m / 2\rfloor}\left(\frac{-1}{4}\right)^{i} \frac{\left(1-t^{2}\right)^{i} t^{m-2 i}}{i !(m-2 i) ! \Gamma\left(i+\frac{d-1}{2}\right)} .
$$

A proof of this theorem can be found in [34]. Especially, for the case $d=3$, the polynomials $P_{m}(3 ; t)$, correspond to the Legendre polynomials, cf. [34].

Notice, that the Funk-Hecke formula applies to all kernel functions on $\mathbb{S}^{d-1}$, which depend only on the Euclidean distance $r(\mathbf{x}, \mathbf{y})=\|\mathbf{x}-\mathbf{y}\|_{2}$. This is easily seen due to $r(\mathbf{x}, \mathbf{y})=r\left(\mathbf{x}^{\top} \mathbf{y}\right)=$ $\sqrt{2-2 \mathbf{x}^{\top} \mathbf{y}}$ for all $\mathbf{x}, \mathbf{y} \in \mathbb{S}^{d-1}$. Figure 2 shows the distribution of the Matern-kernels' eigenvalues

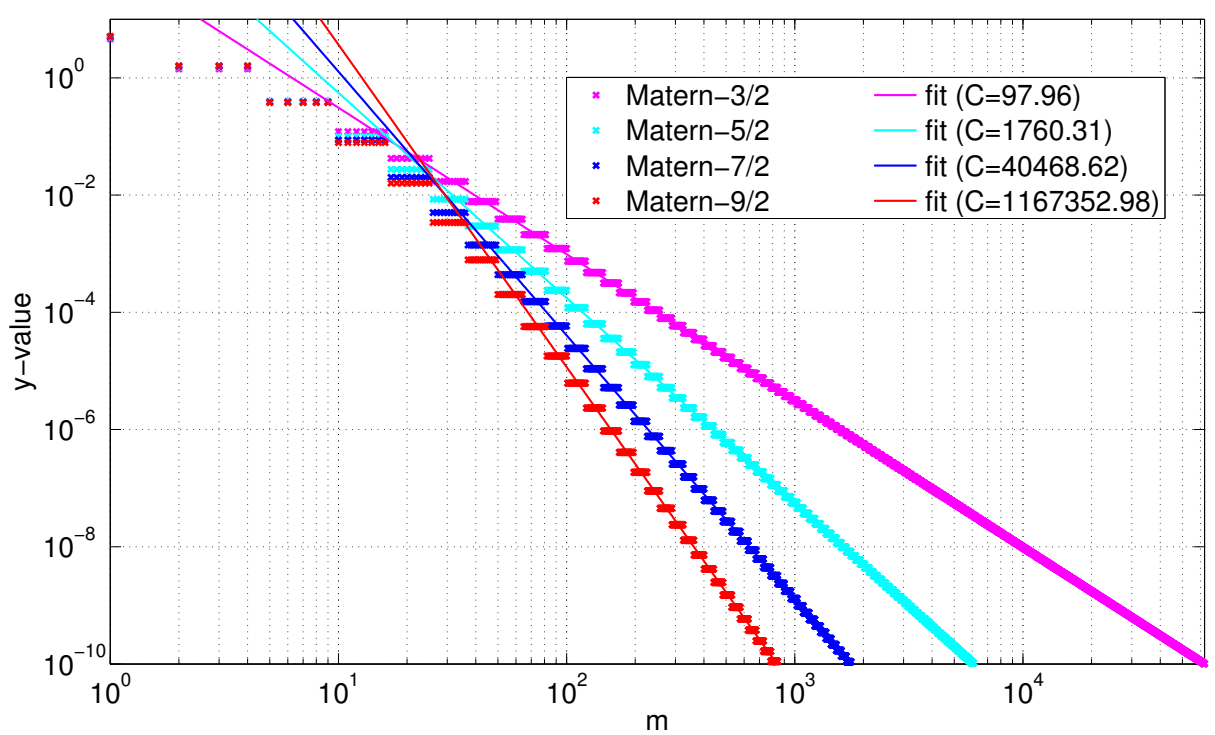

Figure 2. Decay of the eigenvalues with related fits.

for $\nu=3 / 2,5 / 2,7 / 2,9 / 2$ on $\mathbb{S}^{2}$ up to an order of magnitude of $10^{-10}$ for the correlation length 
$\ell=1$. The constant $C$ is estimated by a least-square fit for the ratio of the rate given by formula (22) for $C=1$ and the exact eigenvalues given by Theorem 4.2. The obtained values of $C$ for each kernel under consideration are denoted in the legend of Figure 2. The plot indicates, that the fitted rates perfectly match the asymptotic behavior of the eigenvalues.

\section{NUMERICAL RESULTS}

The numerical tests in this section are performed on parametric surfaces $\Gamma \subset \mathbb{R}^{3}$. These surfaces have recently been considered in the context of solving boundary integral equations, cf. [35] and the references therein. Especially, the implementation of ACA, we apply here, is explained in [35]. The implementations of ACA and PCD rely on the same single-scale code, which means, they use the same quadrature routines for the integration of the Galerkin matrices. In case of ACA, we use ARPACK, cf. [23] to solve the eigen-problem for the covariance operator (5). The size of the Krylov subspace in ARPACK is chosen to twice the number of desired eigenvalues, which is a reasonable choice according to [23]. All computations are carried out on a single core of a computing server with eight Intel(R) Xeon(R) X5550 CPUs with a clock rate of $2.67 \mathrm{GHz}$ and $48 \mathrm{~GB}$ of main memory. Furthermore, we set the correlation length of the Matern kernels to $\ell=1$ in each example.

In the following, let $k_{h, M}(\mathbf{x}, \mathbf{y})$ denote the covariance kernel corresponding to the discretized stochastic field $a_{h, M}(\mathbf{x}, \omega)$ which is either obtained by PCD or by ACA. If the random variables $\left\{X_{m}\right\}_{m}$ are represented exactly in $a_{h, M}(\mathbf{x}, \omega)$, we can measure the error in terms of the (continuous) traces of the related covariance operators. Namely, from [11], we know that

$$
\left\|a-a_{h, M}\right\|_{L^{2}(D) \otimes L_{\mathbb{P}}^{2}(\Omega)} \leq \sqrt{\operatorname{Tr} \mathcal{C}-\operatorname{Tr} \mathcal{C}_{h, M}} .
$$

Denoting the orthonormal eigenfunctions of $\mathcal{C}$ by $\left\{\varphi_{m}\right\}_{m}$, it holds that

$$
\operatorname{Tr} \mathcal{C}=\sum_{m=1}^{\infty}\left(\mathcal{C} \varphi_{m}, \varphi_{m}\right)_{L^{2}(D)}=\sum_{m=1}^{\infty} \lambda_{m}=\int_{D} k(\mathbf{x}, \mathbf{x}) \mathrm{d} \mathbf{x}
$$

by Mercer's theorem. Thus, the trace is easily computable in our application. ${ }^{\dagger}$

\subsection{First example}

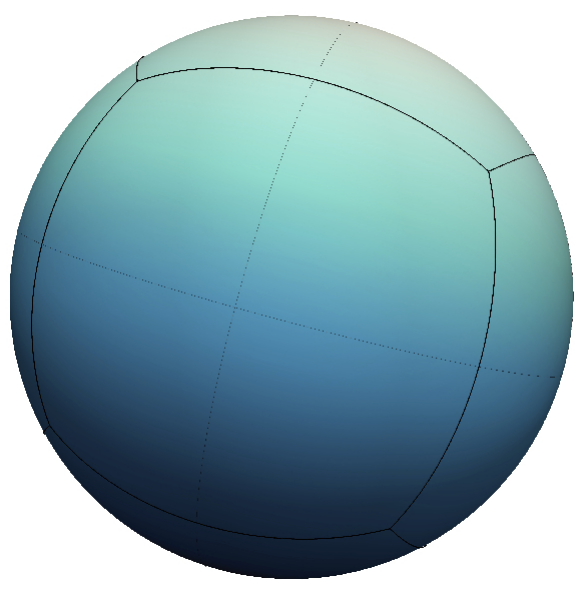

Figure 3. The unit sphere $\mathbb{S}^{2}$ represented by 6 patches.

\footnotetext{
${ }^{\dagger}$ Notice that the Matern kernels are equal to 1 along the diagonal. Therefore, the trace corresponds to the surface measure. Especially, we have $\operatorname{Tr} \mathcal{C}=4 \pi$ on the unit sphere $\mathbb{S}^{2}$ and $\operatorname{Tr} \mathcal{C}=120-7.5 \pi$ for the plate geometry, considered here. 
As a benchmark, we consider the three-dimensional unit sphere $\mathbb{S}^{2} \subset \mathbb{R}^{3}$ represented by 6 congruent patches, see Figure 3. Thus, with the knowledge from the preceding Section 4, we can compute the exact eigenvalues and eigenfunctions of the Matérn covariance functions as reference. Furthermore, we can estimate the truncation error due to (22). For the truncation error related to the Matérn covariance with smoothness parameter $\nu$, it holds that

$$
\sqrt{\sum_{m=M}^{\infty} \lambda_{m}} \lesssim \sqrt{\int_{M}^{\infty} C x^{-1-\nu} \mathrm{d} x}=\sqrt{\frac{1}{\nu} C M^{-\nu}} .
$$

Notice that the dimension is essentially $d=2$ here, since we restrict the Matérn kernels to the unit sphere $\mathbb{S}^{2}$. Using piece-wise constant finite elements, we can achieve a rate of convergence which is proportional to the mesh width $h$. Thus, to bound the truncation error of the Karhunen-Loève expansion by $h$, we have to ensure

$$
\sqrt{\frac{1}{\nu} C M^{-\nu}} \leq h \quad \Rightarrow \quad M \geq\left(\frac{C}{\nu h^{2}}\right)^{\frac{1}{\nu}} .
$$

\begin{tabular}{|r|r|r|r|r|}
\hline$j$ & $\nu=3 / 2$ & $\nu=5 / 2$ & $\nu=7 / 2$ & $\nu=9 / 2$ \\
\hline 1 & $6(9)$ & $4(4)$ & $4(4)$ & $4(4)$ \\
\hline 2 & $18(25)$ & $13(16)$ & $11(16)$ & $9(9)$ \\
\hline 3 & $48(49)$ & $25(25)$ & $20(25)$ & $17(25)$ \\
\hline 4 & $120(121)$ & $45(49)$ & $33(36)$ & $26(36)$ \\
\hline 5 & $305(324)$ & $79(81)$ & $49(49)$ & $40(49)$ \\
\hline 6 & $768(789)$ & $139(144)$ & $76(81)$ & $57(64)$ \\
\hline 7 & $1928(1936)$ & $243(256)$ & $113(121)$ & $78(81)$ \\
\hline 8 & $4807(4900)$ & $423(441)$ & $166(169)$ & $107(121)$ \\
\hline
\end{tabular}

Table I. Different values for the cut-off parameter $M_{j}$ on the unit sphere $\mathbb{S}^{2}$.

\begin{tabular}{|r|r|r|r|r|}
\hline$j$ & $\nu=3 / 2$ & $\nu=5 / 2$ & $\nu=7 / 2$ & $\nu=9 / 2$ \\
\hline 1 & $5(6)$ & $5(6)$ & $4(5)$ & $4(5)$ \\
\hline 2 & $19(21)$ & $14(14)$ & $12(13)$ & $11(12)$ \\
\hline 3 & $49(56)$ & $29(32)$ & $23(24)$ & $21(22)$ \\
\hline 4 & $137(158)$ & $53(58)$ & $38(41)$ & $32(35)$ \\
\hline 5 & $359(414)$ & $97(107)$ & $58(62)$ & $46(49)$ \\
\hline 6 & $935(1082)$ & $167(185)$ & $89(96)$ & $64(69)$ \\
\hline 7 & $2415(2812)$ & $295(327)$ & $132(143)$ & $90(96)$ \\
\hline 8 & $-(7158)$ & $513(569)$ & $197(214)$ & $122(130)$ \\
\hline
\end{tabular}

Table II. Ranks determined by PCD on the unit sphere $\mathbb{S}^{2}$.

With the estimation of the constant $C$ at hand, cf. Figure 2, we could now compute the related length of the Karhunen-Loève expansion. Unfortunately, this approach yields very large numbers of eigen-pairs to be approximated by ACA. Therefore, we choose another approach. We consider for each respective kernel the sum of those eigenvalues with magnitude larger then $10^{-10}$ as an approximation to the actual trace of the kernel, i.e.

$$
\int_{\mathbb{S}^{2}} k_{\nu}(\mathbf{x}, \mathbf{x}) \mathrm{d} s_{\mathbf{x}}=\sum_{m=1}^{M_{\max }} \lambda_{m}+\varepsilon
$$


with $M_{\max }=\arg \min _{m}\left\{\lambda_{m}>10^{-10}\right\}$. The resulting truncation error is computable due to the knowledge of the exact traces which are equal to $4 \pi$ for every $\nu$. We have $\varepsilon=4.18 \cdot 10^{-6}$ for $\nu=3 / 2, \varepsilon=2.43 \cdot 10^{-7}$ for $\nu=5 / 2, \varepsilon=4.93 \cdot 10^{-8}$ for $\nu=7 / 2$, and $\varepsilon=1.70 \cdot 10^{-8}$ for $\nu=9 / 2$. The rank on each level $j$ with mesh-witdh $h=2^{-j}$ is then determined according to

$$
M_{j}=\underset{k \in\left\{1, \ldots, M_{\max }\right\}}{\arg \min }\left\{\sum_{m=1}^{M_{\max }} \lambda_{m}-\sum_{m=1}^{k} \lambda_{m}<h^{2} \sum_{m=1}^{M_{\max }} \lambda_{m}\right\} .
$$

The finest level $j$ which we consider here is 8 , resulting in 393216 finite elements. For the levels $j=1, \ldots, 8$ and $\nu=3 / 2,5 / 2,7 / 2,9 / 2$, the related cut-off parameters $M_{j}$ are found in Table I. The number in the brackets denotes the size necessary to resolve clusters of eigenvalues by approximating only complete subspaces related to the multiplicity of the respective eigenvalue. This is proposed in [23] in order to achieve the optimal performance of ARPACK.

Table II shows the ranks determined by PCD. The numbers in front of the brackets correspond to the recompressed ranks, the numbers within the brackets denote the original rank. As it turns out, the ranks computed by PCD are rather optimal in the sense that they reflect the estimated length of the Karhunen-Loéve expansion determined by formula (22). Especially for increasing smoothness of the kernel function, the determined rank gets successively better.

Remark 5.1. We end up with the spectral decomposition of the approximate covariance $\mathcal{C}_{h, M}$ when we solve the eigen-problem (18) for PCD. By truncating this decomposition with the prescribed relative accuracy $h^{2}$, we achieve an a-posteriori recompression of the PCD. This procedure may at most double the approximation error but reduces the rank by up to $10 \%$ on average in our computations for this article.

The error plots and related computational times for the numerical experiments on the unit sphere are found in Figure 4 and in Figure 5, respectively. Unfortunately, the computations of ACA as well as PCD with recompression for $\nu=3 / 2$ and level 8, i.e. for 393216 finite elements, could not be carried out since the available main memory has been insufficient.

Figure 4 shows the trace error for each particular kernel. The expected rate $2^{-j}$ is indicated in the plots by the dashed black line. The magenta colored line with boxes shows the error for ACA with clusters of eigenvalues resolved, whereas the red line with circles shows the error for ACA with the exact number of eigenvalues computed by (24). The error of the PCD is indicated by the blue lines with squares and finally the error of PCD with recompression is indicated by the cyan colored line with circles. It turns out that all four methods provide the expected rate of convergence in this example. For overview purposes, we have chosen the same colors and markers for each particular method in the subsequent visualizations.

Figure 5 shows the computational times for every method and each particular kernel. There seems to be no significant difference in the times for ACA with clusters of eigenvalues resolved and ACA with the exact number of eigenvalues from (24) for all kernels under consideration. The situation changes if we look at the times for PCD with and without recompression. Especially for $\nu=3 / 2$, the computational time nearly doubles due to the recompression. Thus, the decision if a recompression is reasonable depends on the situation at hand. Nevertheless, we observe that PCD is about a factor of 10 times faster than ACA.

\subsection{Second example}

In our second example, we consider the plate geometry shown in Figure 6. It is a rectangle with 30 inscribed, equi-spaced circular holes, which is represented by 120 patches and scaled to a size of $2 \times 2.4$. Here, the computations are carried out on levels $j=1, \ldots, 6$, where level 6 corresponds to 491520 finite elements. Figure 7 contains a visualization of the four orthonormal eigenfunctions corresponding to the four largest eigenvalues of the Matérn kernel with $\nu=3 / 2$.

In this example, we do not know the number of eigenvalues necessary to achieve the desired precision with ACA and ARPACK. Therefore, we use here the ranks provided by PCD with recompression as reference. The respective values are found in Table III. Again, the numbers in 

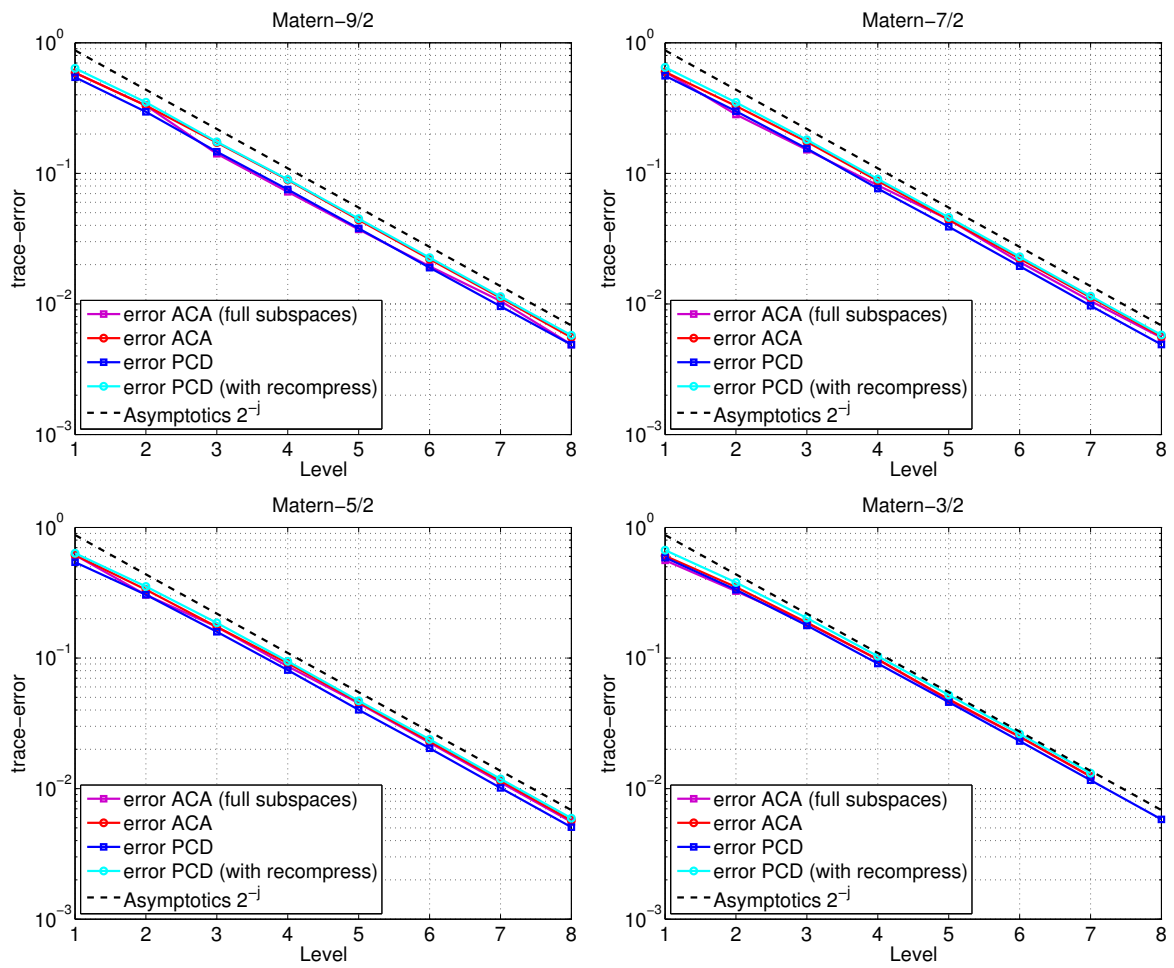

Figure 4. Numerical results (errors) on the unit sphere $\mathbb{S}^{2}$.
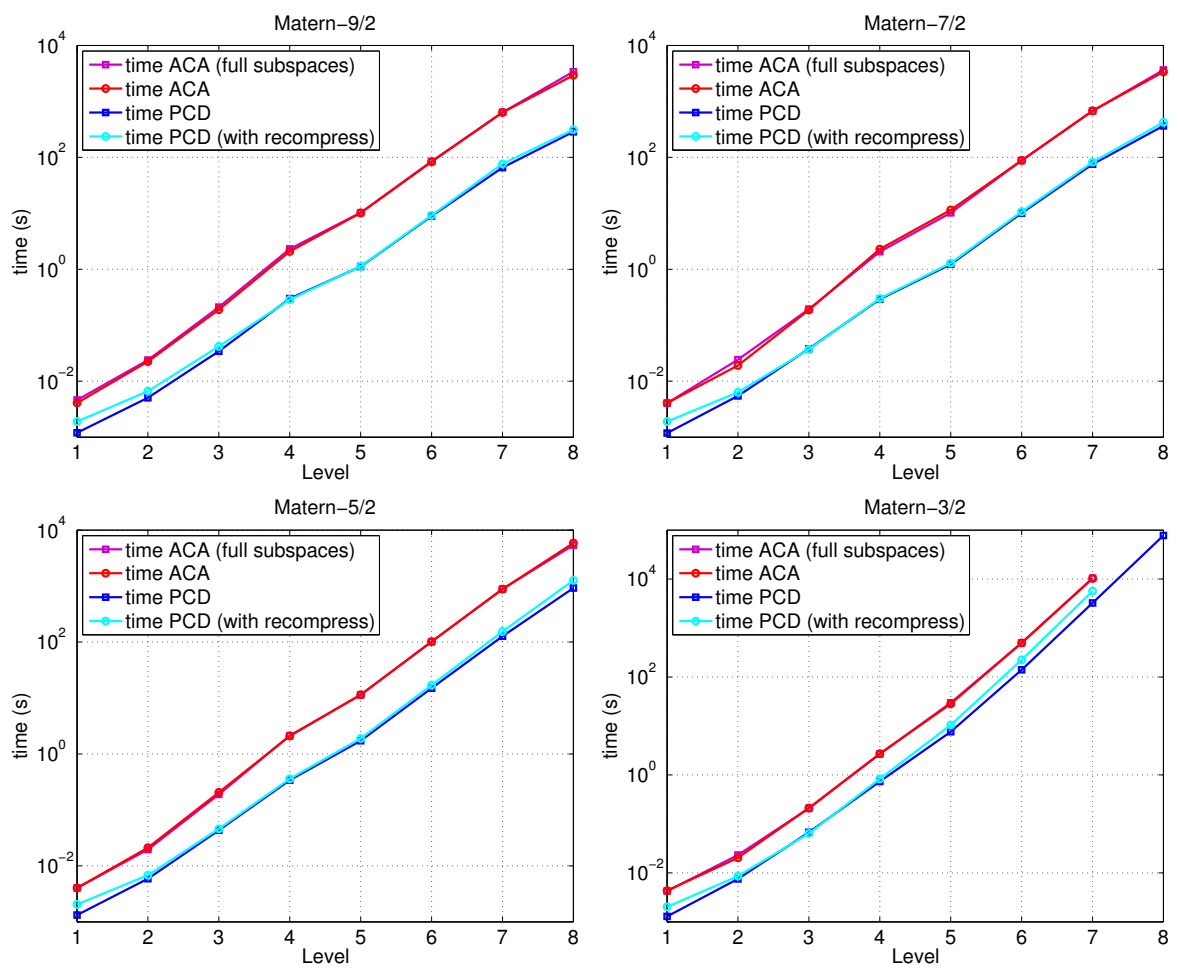

Figure 5. Numerical results (computational times) on the unit sphere $\mathbb{S}^{2}$. 


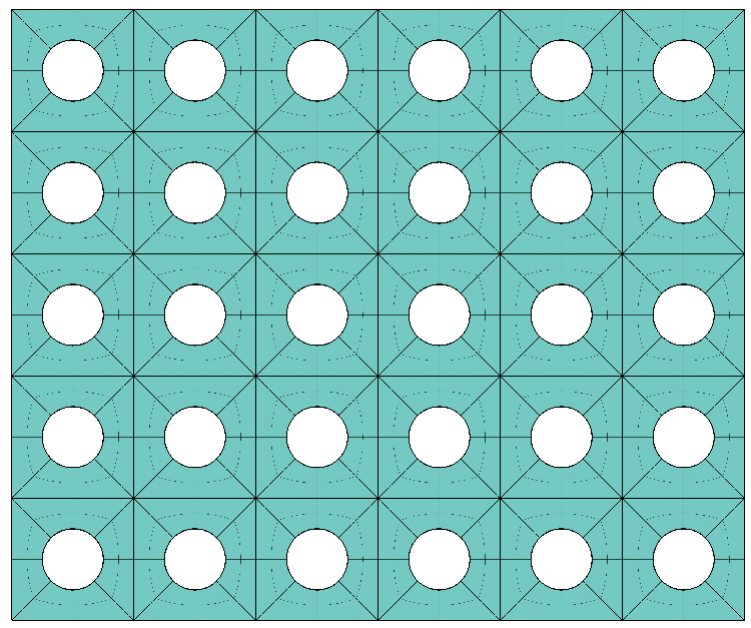

Figure 6. Plate geometry represented by 120 patches.
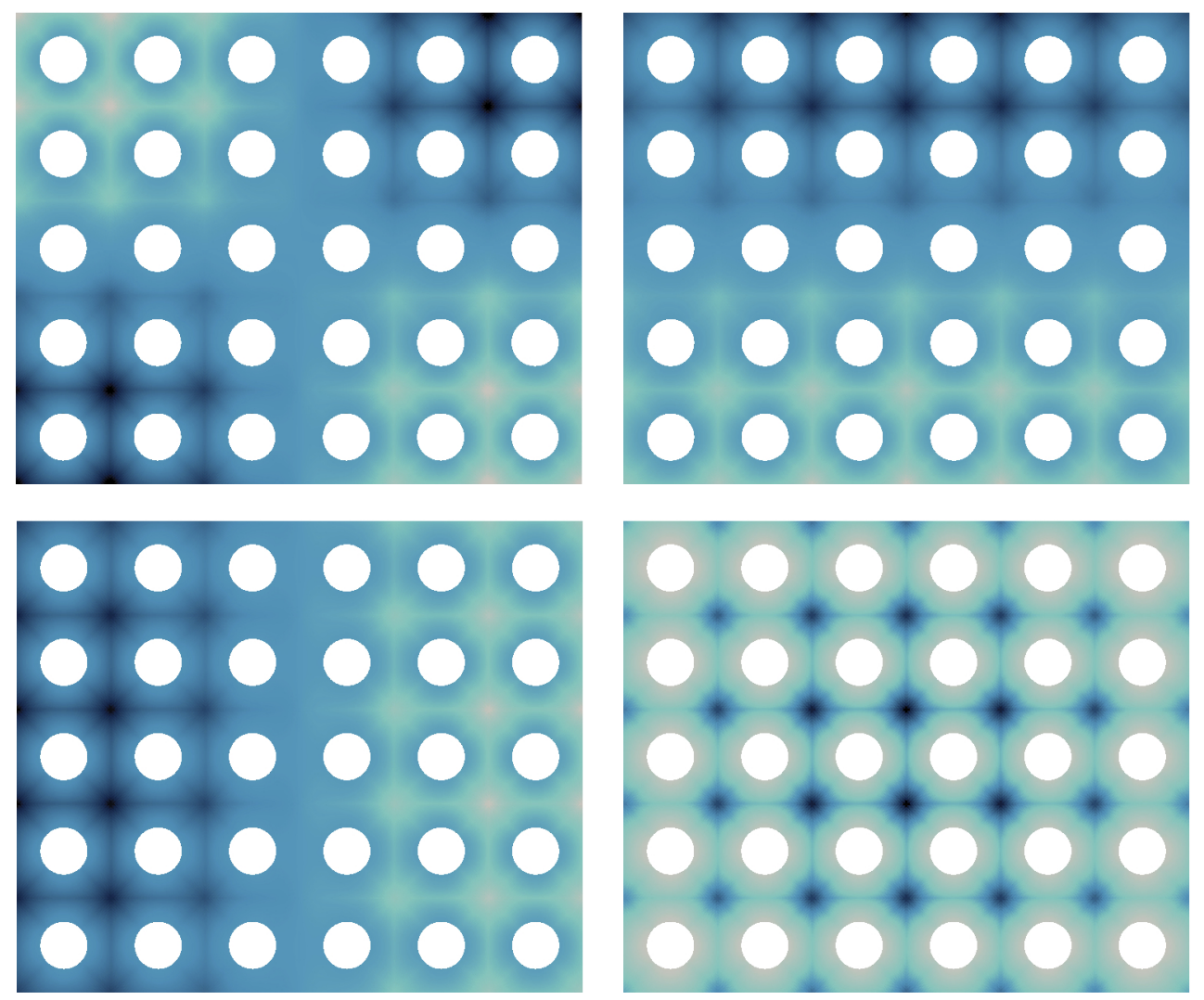

Figure 7. First four orthonormal eigenfunctions on the plate geometry and Matérn kernel for $\nu=3 / 2$.

front of the brackets correspond to the recompressed ranks of PCD and the numbers within the brackets denote the original ranks.

The error plots and related computational times for the numerical experiments on the plate geometry are presented in Figure 8 and in Figure 9, respectively.

The trace error for each particular kernel, i.e. $\nu=3 / 2,5 / 2,7 / 2,9 / 2$, and the different methods is found in Figure 8. Again, PCD provides exactly the expected rate of convergence. Nevertheless, in this regime, PCD with recompression performs from $25 \%$ up to $30 \%$ worse. The behavior of ACA is 


\begin{tabular}{|r|r|r|r|r|}
\hline$j$ & $\nu=3 / 2$ & $\nu=5 / 2$ & $\nu=7 / 2$ & $\nu=9 / 2$ \\
\hline 1 & $13(14)$ & $11(11)$ & $9(9)$ & $9(9)$ \\
\hline 2 & $34(36)$ & $19(20)$ & $15(15)$ & $14(14)$ \\
\hline 3 & $78(86)$ & $31(33)$ & $25(26)$ & $20(21)$ \\
\hline 4 & $178(196)$ & $52(56)$ & $35(37)$ & $29(30)$ \\
\hline 5 & $416(459)$ & $87(93)$ & $49(52)$ & $38(39)$ \\
\hline 6 & $983(1085)$ & $141(151)$ & $71(75)$ & $53(55)$ \\
\hline
\end{tabular}

Table III. Ranks determined by PCD on the plate geometry.
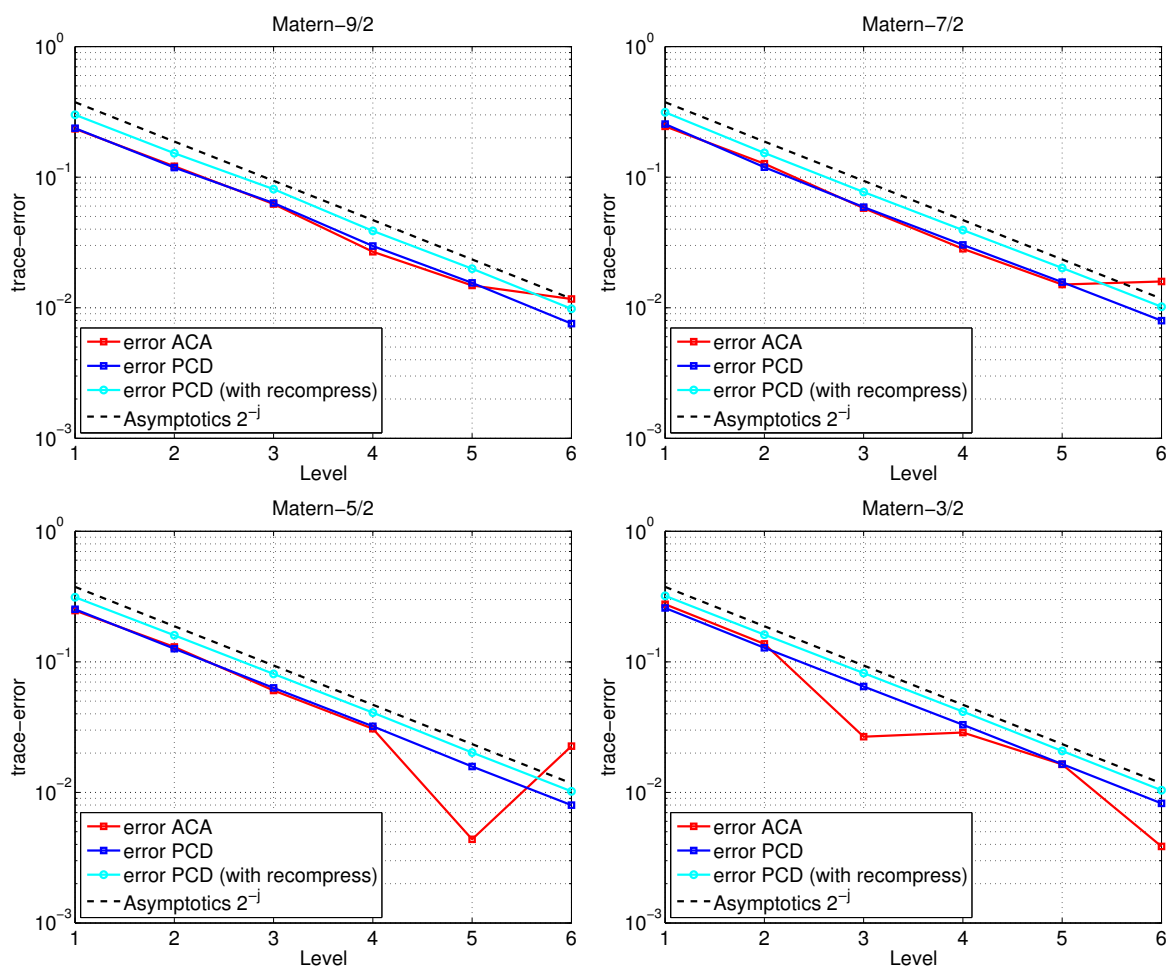

Figure 8. Numerical results (errors) on the plate geometry.

not that monotone as in the previous example. In case of the smoother kernels, i.e. $\nu=7 / 2,9 / 2$, the rate of convergence deteriorates in the last step. For $\nu=3 / 2$ we have a contrary behavior. The rate of convergence is increased from level 2 to 3 and in the last step. Finally, we observe for $\nu=5 / 2$ an increased rate of convergence from level 4 to 5 on the one hand and an increase of the error in the last step on the other hand. Possibly, these effects are caused by a lack of resolution of the faster oscillating eigenfunctions which are involved in the deflation process of the implicit restarted Arnoldi method and the resulting impact on the computation of the sought eigenvalues.

Figure 9 shows the computational times for every method and each particular kernel. Here, the times for the recompression of PCD are rather moderate due to the low ranks. Nevertheless, the benefit of the recompression is relatively small here, especially for the smoother kernels, cf. Table III. Again, PCD outperforms ACA by about a factor of 10 . 

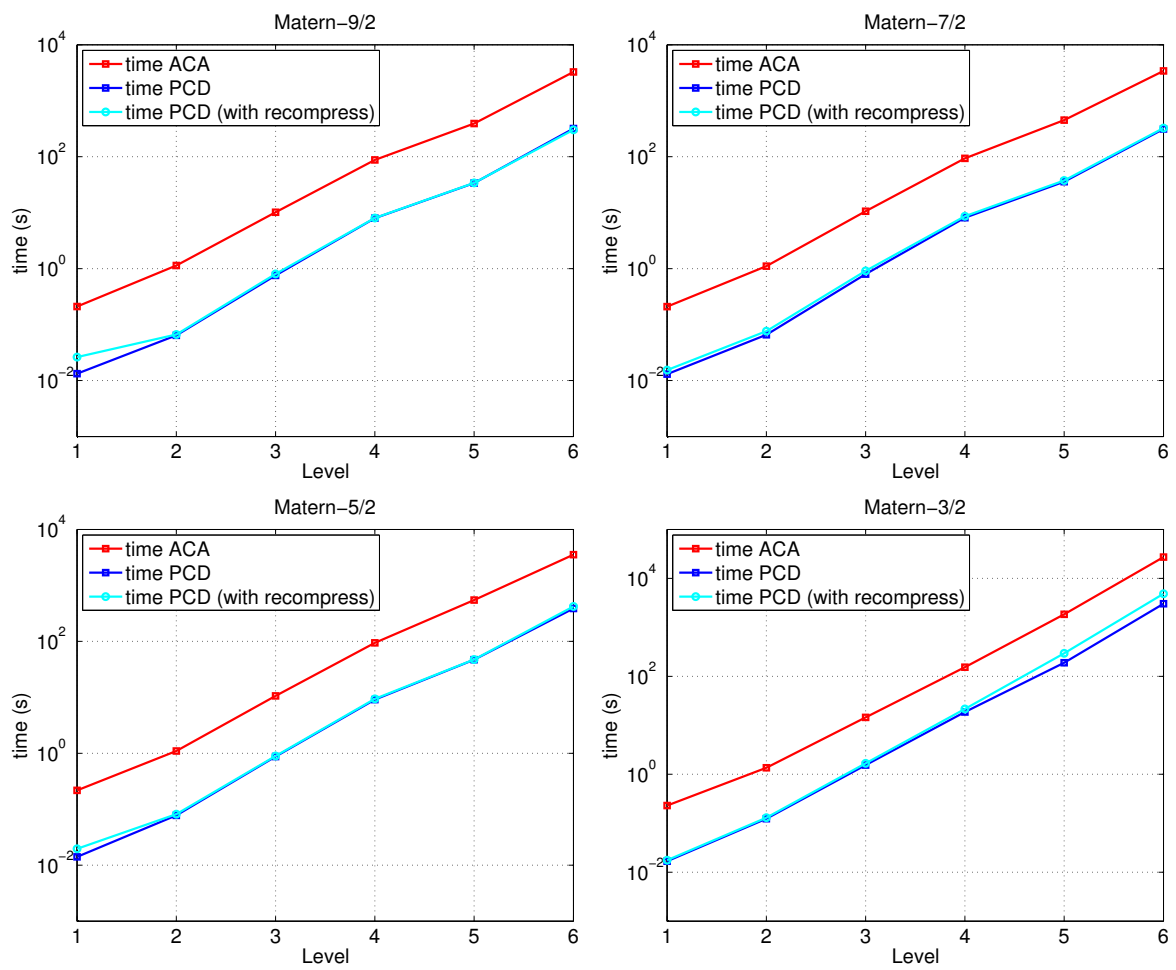

Figure 9. Numerical results (computational times) on the plate geometry.

\section{CONCLUDING REMARKS}

The present article is devoted to the efficient approximation of random fields for numerical applications. It is state of the art to compute a separable representation of the random field under consideration. A very common approach to determine such a representation is the (truncated) Karhunen-Loève expansion. Here, one has to solve the eigen-problem for the related covariance operator. We have tackled this task by combining the Adaptive Cross Approximation and ARPACK. Nevertheless, a major drawback of this approach is that the number of eigen-pairs to be approximated has to be known in advance. This might be a problem in practice since the correct number is not feasible in many applications. With the pivoted Cholesky decomposition, we provide a method which overcomes this obstruction. Due to the knowledge of the discretized covariance operator's main diagonal, we are able to a-posteriori control the approximation error in terms of the trace norm. If, for the application at hand, an orthogonal decomposition of the stochastic field is required, this can be realized relatively cheap by the pivoted Cholesky decomposition in a postprocessing step. The numerical experiments suggest that both approaches provide the optimal rate of convergence. In the comparison of the computational times, we observe however that the pivoted Cholesky decomposition is the superior method.

\section{REFERENCES}

1. Ghanem R, Spanos P. Stochastic finite elements. A spectral approach. Springer: New York, 1991.

2. Rasmussen CE, Williams CKI. Gaussian Processes for Machine Learning (Adaptive Computation and Machine Learning). The MIT Press: Cambridge, 2005.

3. Babuška I, Nobile F, Tempone R. A stochastic collocation method for elliptic partial differential equations with random input data. SIAM Journal on Numerical Analysis 2007; 45(3):1005-1034.

4. Babuška I, Tempone R, Zouraris G. Galerkin finite element approximations of stochastic elliptic partial differential equations. SIAM Journal on Numerical Analysis 2004; 42(2):800-825. 
5. Frauenfelder P, Schwab C, Todor R. Finite elements for elliptic problems with stochastic coefficients. Computer Methods in Applied Mechanics and Engineering 2005; 194(2-5):205-228.

6. Matthies H, Keese A. Galerkin methods for linear and nonlinear elliptic stochastic partial differential equations. Computer Methods in Applied Mechanics and Engineering 2005; 194(12-16):1295-1331.

7. Schwab C, Gittelson CJ. Sparse tensor discretizations of high-dimensional parametric and stochastic PDEs. Acta Numerica 2011; 20:291-467.

8. Light WA, Cheney EW. Approximation theory in tensor product spaces. Lecture notes in mathematics, Springer: Berlin-Heidelberg, 1985.

9. Loève M. Probability theory. I+II. Fourth edn., no. 45 in Graduate Texts in Mathematics, Springer: New York, 1977.

10. Giebermann K. Multilevel approximation of boundary integral operators. Computing 2001; 67(3):183-207.

11. Schwab C, Todor R. Karhunen-Loève approximation of random fields by generalized fast multipole methods. Journal of Computational Physics 2006; 217:100-122.

12. Hackbusch W. Hierarchische Matrizen: Algorithmen und Analysis. Springer: Heidelberg, 2009.

13. Eiermann M, Ernst OG, Ullmann E. Computational aspects of the stochastic finite element method. Computing and Visualization in Science 2007; 10(1):3-15.

14. Saad Y. Numerical Methods for Large Eigenvalue Problems. Manchester University Press: Manchester, 1992.

15. Bebendorf M, Rjasanow S. Adaptive low-rank approximation of collocation matrices. Computing 2003; 70(1):124.

16. Bebendorf M. Approximation of boundary element matrices. Numerische Mathematik 2000; 86(4):565-589.

17. Dahmen W, Harbrecht H, Schneider R. Compression techniques for boundary integral equations. Asymptotically optimal complexity estimates. SIAM Journal on Numerical Analysis 2006; 43(6):2251-2271.

18. Harbrecht H, Schneider R. Wavelet galerkin schemes for boundary integral equations. Implementation and quadrature. SIAM Journal on Scientific Computing 2006; 27(4):1347-1370.

19. Harbrecht H, Peters M, Schneider R. On the low-rank approximation by the pivoted Cholesky decomposition. Applied Numerical Mathematics 2012; 62:28-440.

20. Sauter SA, Schwab C. Quadrature for hp-Galerkin BEM in $\mathbb{R}^{3}$. Numerische Mathematik 1997; 78(2):211-258.

21. Golub GH, Van Loan CF. Matrix Computations. Fourth edn., Johns Hopkins University Press: Baltimore, 2012.

22. Lehoucq RB, Sorensen DC. Deflation techniques for an implicitly restarted Arnoldi iteration. SIAM Journal on Matrix Analysis and Applications 1996; 17(4):789-821.

23. Lehoucq RB, Sorensen DC, Yang C. Arpack User's Guide: Solution of Large-Scale Eigenvalue Problems With Implicityly Restorted Arnoldi Methods (Software, Environments, Tools). SIAM: Philadelphia, 1998.

24. Sorensen DC. Implicit application of polynomial filters in a k-step Arnoldi method. SIAM Journal on Matrix Analysis and Applications 1992; 13(1):357-385.

25. Diestel J, Uhl JJ. Vector Measures. Mathematical surveys and monographs, American Mathematical Society, 1977.

26. Braess D. Finite Elements. Theory, fast solvers, and applications in solid mechanics. Second edn., Cambridge University Press: Cambridge, 2001.

27. Brenner S, Scott L. The mathematical theory of finite element methods. Third edn., Springer: Berlin, 2008.

28. Griebel M, Harbrecht H. Approximation of bi-variate functions: singular value decomposition versus sparse grids. IMA Journal of Numerical Analysis 2014; To appear.

29. D’yakonov EG, McCormick SF. Optimization in Solving Elliptic Problems. CRC Press: Boca Raton, 1996.

30. Hammarling S, Higham NJ, Lucas C. LAPACK-style codes for pivoted cholesky and QR updating. Applied Parallel Computing. State of the Art in Scientific Computing, Kågström B, Elmroth E, Dongarra J, Waśniewski J (eds.). No. 4699 in Lecture Notes in Computer Science, Springer: Berlin-Heidelberg, 2007; 137-146.

31. Matern B. Spatial Variation. Springer Lecture Notes in Statistics, Springer: New York, 1986.

32. Abramowitz M, Stegun IA. Handbook of Mathematical Functions: With Formulas, Graphs, and Mathematical Tables. Applied mathematics series, Dover Publications, 1964.

33. Graham I, Kuo F, Nichols J, Scheichl R, Schwab C, Sloan I. Quasi-Monte Carlo finite element methods for elliptic PDEs with log-normal random coefficient. SAM-Report 2013; 2013-14.

34. Müller C. Analysis of Spherical Symmetries in Euclidean Spaces. No. 129 in Applied Mathematical Sciences Series, Springer: Berlin-Heidelberg, 1998.

35. Harbrecht H, Peters M. Comparison of fast boundary element methods on parametric surfaces. Computer Methods in Applied Mechanics and Engineering 2013; 261-262:39-55. 


\section{LATEST PREPRINTS}

No. Author: Title

2013-18 Helmut Harbrecht, Michael Peters, Markus Siebenmorgen

Multilevel Accelerated Quadrature for PDEs with Log-Normal Distributed Random Coefficient*

2013-19 Jérémy Blanc, Serge Cantat

Dynamical Degrees of Birational Transformations of Projective Surfaces

2013-20 Jérémy Blanc, Frédéric Mangolte

Cremona Groups of Real Surfaces

2013-21 Jérémy Blanc, Igor Dolgachev

Automorphisms of Cluster Algebras of Rank 2

2013-22 Helmut Harbrecht, Giannoula Mitrou

Improved Trial Methods for a Class of Generalized Bernoulli Problems

2013-23 Helmut Harbrecht, Johannes Tausch

On Shape Optimization with Parabolic State Equation

2013-24 Zoé Chatzidakis, Dragos Ghioca, David Masser, Guillaume Maurin Unlikely, Likely and Impossible Intersections without Algebraic Groups

2013-25 Assyr Abdulle, Marcus J. Grote, Christian Stohrer

Finite Element Heterogeneous Multiscale Method for the Wave Equations : Long Time Effects

2013-26 Luigi Ambrosio and Gianluca Crippa

Continuity Equations and ODE Flows with Non-Smooth Velocity

2013-27 Andriy Regeta

Lie Subalgebras of Vector Fields and the Jacobian Conjecture

2013-28 Helmut Harbrecht, Michael Peters, Markus Siebenmorgen

Tractability of the Quasi-Monte Carlo Quadrature with Halton Points for Elliptic Pdes with Random Diffusion

2013-29 Helmut Harbrecht, Giannoula Mitrou

Stabilization of the Trial Method for the Bernoulli Problem in Case of Prescribed Dirichlet Data

2014-01 Helmut Harbrecht, Michael Peters, Markus Siebenmorgen

Efficient Approximation of Random Fields for Numerical Applications 